ISSN 1855-3966 (printed edn.), ISSN 1855-3974 (electronic edn.)

\author{
ARS MATHEMATICA CONTEMPORANEA 22 (2022) \#P3.04 \\ https://doi.org/10.26493/1855-3974.2134.ac9 \\ (Also available at http://amc-journal.eu)
}

\title{
Convex drawings of the complete graph: topology meets geometry*
}

\author{
Alan Arroyo ${ }^{\dagger}$ \\ University of Waterloo, Waterloo, Ontario, Canada, \\ current address: Vienna, Austria \\ Dan McQuillan \\ Norwich University, Northfield, Vermont, United States \\ R. Bruce Richter $\stackrel{0}{\ddagger}$ \\ University of Waterloo, Waterloo, Ontario, Canada \\ Gelasio Salazar ${ }^{\S}$ \\ Universidad Autónoma de San Luis Potosí, Mexico
}

Received 1 October 2019, accepted 8 September 2021, published online 24 June 2022

\begin{abstract}
In a geometric drawing of $K_{n}$, trivially each 3-cycle bounds a convex region: if two vertices are in that region, then so is the (geometric) edge between them. We define a topological drawing $D$ of $K_{n}$ in the sphere to be convex if each 3-cycle bounds a closed region $R$ (either of the two sides of the 3-cycle) such that any two vertices in $R$ have the (topological) edge between them contained in $R$.

While convex drawings generalize geometric drawings, they specialize topological ones. Therefore it might be surprising if all optimal (that is, crossing-minimal) topological drawings of $K_{n}$ were convex. However, we take a first step to showing that they are convex: we show that if $D$ has a non-convex $K_{5}$ all of whose extensions to a $K_{7}$ have no other non-convex $K_{5}$, then $D$ is not optimal (without reference to the conjecture for the crossing number of $K_{n}$ ). This is the first example of non-trivial local considerations providing sufficient conditions for suboptimality. At our request, Aichholzer has computationally verified that, up to $n=12$, every optimal drawing of $K_{n}$ is convex.
\end{abstract}

\footnotetext{
*We are very grateful to the referees for their detailed reading and useful suggestions for some simplifications and improving presentation. We also appreciate the contributions of Matthew Sullivan and Kasper Szabo Lyngsie for simplifying some of our original arguments.

${ }^{\dagger}$ Supported by CONACYT.

$\$$ Corresponding author. Supported by NSERC grant \#50503-10940-500.

${ }^{\S}$ Supported by CONACYT.
} 
Convexity naturally lends itself to refinements, including hereditarily convex (h-convex) and face convex (f-convex). The hierarchy rectilinear $\subseteq$ f-convex $\subseteq$ h-convex $\subseteq$ convex $\subseteq$ topological provides links between geometric and topological drawings. It is known that $\mathrm{f}$-convex is equivalent to pseudolinear (generalizing rectilinear) and h-convex is equivalent to pseudospherical (generalizing spherical geodesic). We characterize h-convexity by three forbidden (topological) subdrawings.

This hierarchy provides a framework to consider generalizations of other geometric questions for point sets in the plane. We provide two examples of such questions, namely numbers of empty triangles and existence of convex $k$-gons.

Keywords: Simple drawings, complete graphs, convex drawings.

Math. Subj. Class. (2020): 05C10

\section{Introduction}

Hill's long-standing conjecture [14, 10] asserts that the crossing number $\operatorname{cr}\left(K_{n}\right)$ of $K_{n}$ is equal to

$$
H(n):=\frac{1}{4}\left\lfloor\frac{n}{2}\right\rfloor\left\lfloor\frac{n-1}{2}\right\rfloor\left\lfloor\frac{n-2}{2}\right\rfloor\left\lfloor\frac{n-3}{2}\right\rfloor .
$$

To date, Hill's conjecture has only been verified up to $n \leq 12$. Moreover, current proofs for $n=11,12$ rely on extensive computer searches, therefore providing limited explanation for the elegance of the expression in Hill's conjecture. Guy's [13] original proof for $n=9,10$ also relied on an extensive case analysis, with most details left to the reader, similar to a computer proof.

The main point of this work is the introduction of the class of convex drawings of $K_{n}$. It turns out that, of the (up to spherical homeomorphisms) five drawings of $K_{5}$ in the sphere, the drawings $\widetilde{\mathbb{K}}_{5}^{3}$ and $\widetilde{\mathbb{K}}_{5}^{5}$ in Figure 1 are not convex in our sense. Furthermore, an elementary but principal result of this work is to characterize (rather than define) a spherical drawing of $K_{n}$ as convex if and only if neither $\widetilde{\mathbb{K}}_{5}^{3}$ nor $\widetilde{\mathbb{K}}_{5}^{5}$ occurs as a subdrawing.

Our study of these drawings was motivated by a couple of specific events. One was the computer-free proof by two of the authors that the crossing number of $K_{9}$ is 36 [21]. As part of that proof, the two drawings $\widetilde{\mathbb{K}}_{5}^{3}$ and $\widetilde{\mathbb{K}}_{5}^{5}$ were both shown not to occur in any optimal (that is, fewest crossings) drawing of $K_{7}$. Another was the question, "Is there, for some $n$, an optimal drawing (or even one with the conjectured fewest crossings) of $K_{n}$ that contains $\widetilde{\mathbb{K}}_{5}^{5}$ ?"

One of us asked Tilo Wiedera to check by computer if any optimal drawing of $K_{9}$ contains a $\widetilde{\mathbb{K}}_{5}^{5}$. Not only was the answer negative as expected, but Wiedera also found that the smallest number of crossings in a drawing of $K_{9}$ that contains $\widetilde{\mathbb{K}}_{5}^{5}$ has 40 crossings a surprising 4 more than optimal! At the Crossing Number Workshop in Osnäbruck (May 2017), the authors then asked Aichholzer for the smallest $n$ for which an optimal drawing of $K_{n}$ could contain it. After checking for both $\widetilde{\mathbb{K}}_{5}^{3}$ and $\widetilde{\mathbb{K}}_{5}^{5}$ among all optimal drawings for $K_{n}$ with $n \leq 12$, he announced at the workshop his findings, implying that if such $n$ exists, it must be at least 13 . Theorem 5.1 below gives further evidence that the answer to the question is "no".

E-mail addresses: alanarroyoguevara@gmail.com (Alan Arroyo),dmcquill@ norwich.edu (Dan McQuillan), brichter@uwaterloo.ca (R. Bruce Richter), gsalazar@ifisica.uaslp.mx (Gelasio Salazar) 

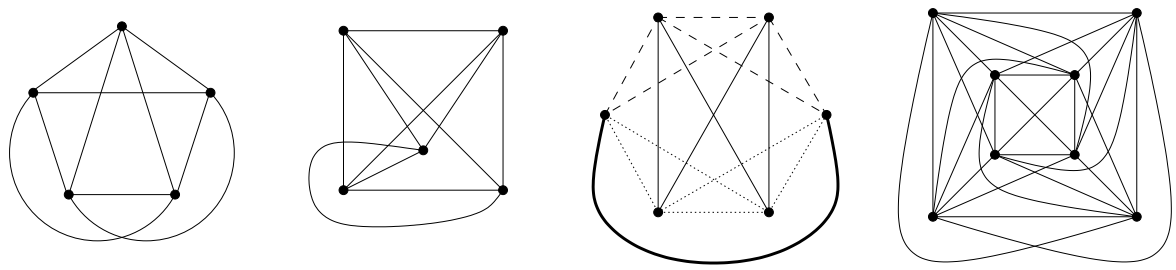

Figure 1: Drawings of interest: $\widetilde{\mathbb{K}}_{5}^{3}, \widetilde{\mathbb{K}}_{5}^{5}, \mathbb{K}_{6}^{11}$, and $T C_{8}$.

Thus, we were quite naturally led to the class of simple (i.e., no edge crosses itself and no two closed edges intersect twice; often referred to as "good") drawings of $K_{n}$ in which neither $\widetilde{\mathbb{K}}_{5}^{3}$ nor $\widetilde{\mathbb{K}}_{5}^{5}$ occurs; we first thought of these as "locally rectilinear", as, of the (up to spherical homeomorphisms) five drawings of $K_{5}$ in the sphere, these are the two that are not isomorphic (via a homeomorphism from the sphere with an appropriate point deleted to the plane) to rectilinear drawings of $K_{5}$. We became especially interested in them when we realized they have a topological characterization (Theorem 2.6, below), which we have since taken to be the definition of convex drawings in Definition 1.1 below.

If $D$ is a drawing of a graph $G$, and $H$ is a subgraph of $G$ (or even a set of vertices and edges of $G$ ), then we let $D[H]$ denote the drawing of $H$ induced by $D$. In a simple drawing $D$ of a graph $G$, for a 3-cycle $T$ of $G, D[T]$ is a simple closed curve.

Definition 1.1. Let $D$ be a simple drawing of $K_{n}$ in the sphere.

1. If $T$ is a 3-cycle in $K_{n}$, then a closed disc $\Delta$ bounded by $D[T]$ is a convex side of $T$ if, for any distinct vertices $x$ and $y$ of $K_{n}$ such that $D[x]$ and $D[y]$ are both contained in $\Delta$, then $D[x y]$ is also contained in $\Delta$.

2. The drawing $D$ is convex if every 3 -cycle of $K_{n}$ has a convex side.

Evidently every rectilinear or spherical geodesic drawing is convex. Therefore, the "tin can" drawing $T C_{8}$ of $K_{8}$ shown in Figure 1 is convex (see Section 2); it is not homeomorphic to any rectilinear drawing. Indeed, $K_{8}$ is known to have rectilinear crossing number of 19 , while $T C_{8}$ is optimal with the minimum of 18 crossings.

Wagner [25] showed that establishing the Hill Conjecture for spherical geodesic drawings is equivalent to the special case of the Spherical Generalized Upper Bound Conjecture for arrangements of $n$ hemispheres in an $(n-4)$-dimensional sphere. In particular, proving the convex or h-convex crossing number of $K_{n}$ is $H(n)$ would establish this case of the SGUBC. Flag algebras are used by Balogh et al. [7] to show that $\lim _{n \rightarrow \infty} \operatorname{cr}\left(K_{n}\right) / H(n)>0.985$. Restricted to convex drawings, the same technique gets a lower bound of 0.996 . The 0.996 is also the lower bound for spherical geodesic drawings (these are all necessarily convex).

There are two natural refinements of Definition 1.1; the first is satisfied by spherical geodesic drawings, while the second holds for rectilinear and pseudolinear drawings.

Definition 1.2. Let $D$ be a convex drawing of $K_{n}$.

1. Then $D$ is hereditarily convex (abbreviated to h-convex) if, for every 3 -cycle $T$, there is a choice $\Delta_{T}$ of a convex side, such that, if $T_{1}$ and $T_{2}$ are 3-cycles with $D\left[T_{2}\right] \subseteq \Delta_{T_{1}}$, then $\Delta_{T_{2}} \subseteq \Delta_{T_{1}}$. 
2. Then $D$ is face convex (abbreviated to f-convex) if there is a face $\Gamma$ of $D$ such that, for every 3 -cycle $T$ of $K_{n}$, the side of $D[T]$ disjoint from $\Gamma$ is convex.

The drawing $\mathbb{K}_{6}^{11}$ of $K_{6}$ shown in Figure 1 is convex but not h-convex. The (optimal) drawing $T C_{8}$ of $K_{8}$ is h-convex but not f-convex.

It is an easy exercise to prove that an f-convex drawing is also h-convex. Moreover, every rectilinear (or, more generally, pseudolinear) drawing of $K_{n}$ is f-convex, with (in both cases) $\Gamma$ being the unbounded face. In fact, Aichholzer et al. [4] and, independently, the current authors [5], have shown that f-convex is equivalent to pseudolinear. Generalizing spherical geodesic drawings, Arroyo et al. [6] have introduced a natural notion of "pseudospherical drawings" of $K_{n}$ in the sphere; surprisingly, they are exactly the h-convex drawings.

It would be very interesting to obtain an analogous "geometric-style" generalization that characterizes convexity. At this time, we have no suggestion for what this might be.

Thus, our definitions regarding drawings of complete graphs correspond precisely to geometric descriptions of point-sets. These geometric connections open up new possibilities for studying geometric questions to see how the results differ for convex drawings. For complete graphs, we now have a geometrically meaningful hierarchy of drawings. It is, from most to least restrictive: rectilinear $\subseteq$ f-convex (= pseudolinear) $\subseteq$ h-convex (= pseudospherical $\subseteq$ convex $\subseteq$ topological.

One question of long-standing interest is: given $n$ points in general position in the plane, how many of the 3-tuples (that is, triangles) have none of the other points inside the triangle (empty triangle)? Currently, we know that there can be as few as about $1.6 n^{2}+\mathrm{o}\left(n^{2}\right)$ empty triangles [9] and every set of $n$ points has at least $n^{2}+O(n)$ empty triangles $\left(n^{2}+o\left(n^{2}\right)\right.$ first proved in [8]). In [5], we proved the $n^{2}+o\left(n^{2}\right)$ bound also holds for f-convex drawings. At the other extreme, Harborth [15] presented an example of a topological drawing of $K_{n}$ having only $2 n-4$ empty triangles, while Aichholzer et al. [3] show that every topological drawing of $K_{n}$ has at least $n$ empty triangles. We have shown in [5] that every convex drawing of $K_{n}$ has at least $\frac{1}{3} n^{2}+O(n)$ empty triangles. For h-convex, it is shown in [6], using the f-convex result and other facts about h-convex drawings, that there are at least $\frac{3}{4} n^{2}+o\left(n^{2}\right)$ empty triangles. We would be interested in progress related to the coefficients $\frac{1}{3}$ and $\frac{3}{4}$.

Another question of interest is: given $n$ points in general position in the plane, what is the largest $k$ so that $k$ of the $n$ points are the corners of a $k$-gon in convex position? In Theorem 3.4, we generalize to convex drawings the Erdôs-Szekeres theorem [12] that, for every $k$, there is an $n$ such that every set of $n$ points in the plane in general position has a set of $k$ points that are the corners of a convex $k$-gon. Finding the least such $n$ is of current interest. Suk [24] has shown that $2^{k+\mathrm{o}(k)}$ points suffices in the geometric case. For $k=5$, 9 points is best possible in the rectilinear case (see Bonnice [11] for a short proof).

For a general drawing $D$ of $K_{n}$, we can ask whether there is a subdrawing $D\left[K_{k}\right]$ such that one face is bounded by a $k$-cycle: this is a natural drawing of $K_{k}$. (In [21], these drawings are quite appropriately labelled "convex". We think convex is very descriptive of the drawings considered in this work and expect there to be no confusion with the two quite different uses of the term "convex".) Bonnice's proof adapts easily to the pseudolinear case (that is, the f-convex case). Aichholzer (personal communication) has verified by computer that 11 points is best possible for $k=5$ for the convex drawings considered in this article. A trivial consequence of our Theorem 4.5 characterizing h-convex drawings is that any convex, but not h-convex, drawing has a natural $K_{5}$; therefore, 11 is also best possible 
for h-convex drawings. For general drawings of $K_{n}$, there need not be a natural $K_{5}$. In Harborth's example [15] (originally from [16]), every $K_{5}$ is isomorphic to $\widetilde{\mathbb{K}}_{5}^{5}$.

We remark that Pach et al. [23] show that, for any fixed positive integer $r$, there is a large enough integer $N=N(r)$ such that, for $n \geq N$, every simple drawing of $K_{n}$ contains either a natural $K_{r}$ or Harborth's generalization of $\widetilde{\mathbb{K}}_{5}^{5}$ on $r$ vertices. In Harborth's generalization, every $K_{5}$ is isomorphic to $\widetilde{\mathbb{K}}_{5}^{5}$. These are the "twisted" $K_{r}$ s in [23].

Section 2 introduces many fundamental properties of a convex drawing $D$ of $K_{n}$, including showing convexity of $D$ is equivalent to not containing either of the two nonrectilinear drawings $\widetilde{\mathbb{K}}_{5}^{3}$ and $\widetilde{\mathbb{K}}_{5}^{5}$ of $K_{5}$ (the first two drawings in Figure 1). Another equivalence is that every 3 -cycle $T$ has a side such that every vertex $v$ on that side is such that $D[T+v]$ is a non-crossing $K_{4}$.

Section 3 proves that a convex drawing $D$ of $K_{n}$ has a particularly nice structure: there is a natural $K_{r}$ such that $D\left[K_{r}\right]$ has a face $\Gamma$ bounded by an $r$-cycle $C$; if $D[v]$ is in $\Gamma$ and $D[w]$ is in the closure of $\Gamma$, then $D[v w]$ is in $\Gamma \cup\{D[w]\}$; and if $D[v]$ and $D[w]$ are in the closure of the complement of $\Gamma$, then $D[v w]$ is in the complement of $\Gamma$. This structure theorem may provide a strategy for showing that a convex drawing of $K_{n}$ has at least $H(n)$ crossings.

Section 4 treats h-convex drawings. The main result here is that a convex drawing $D$ is h-convex if and only if there is no $K_{6}$ such that $D\left[K_{6}\right]$ is $\mathbb{K}_{6}^{11}$ in Figure 1 . We do not know a comparable result distinguishing f-convex drawings from h-convex. The tin can drawing $T C_{8}$ of $K_{8}$ in Figure 1 is one such (as are the larger tin can drawings). However it is not clear to us whether $T C_{8}$ is the only minimal one or, indeed, if there are only finitely many minimal distinguishing examples. The final result of the section is that testing a set of convex sides for h-convexity is also a "Four Point Property", which is to say that it can be verified by checking all sets of four points.

Finally, in Section 5, we prove the principal result Theorem 5.1, showing that some non-convex drawings of $K_{n}$ are not optimal.

Table 1: The convexity hierarchy.

\begin{tabular}{|c|c|c|}
\hline level & characterization & distinguish \\
\hline general & edges share $\leq 1$ point & \\
\hline convex & general, no $\widetilde{\mathbb{K}}_{5}^{3}, \widetilde{\mathbb{K}}_{5}^{5}$ & $\widetilde{\mathbb{K}}_{5}^{3}$ \\
\hline h-convex & convex, no $\mathbb{K}_{6}^{11}$ & $\mathbb{K}_{6}^{11}$ \\
\hline f-convex & h-convex + ?? & $T C_{8}$ \\
\hline rectilinear & unlikely & Pappus \\
\hline
\end{tabular}

This work will provide characterizations of the different kinds of convexity and distinguishing between them by examples and theorems. A summary is given in Table 1.

Matoušek [19] gives a nice exposition of the theorem of Mnëv [22] that testing the stretchability of an arrangement of pseudolines in the plane is $\exists \mathbb{R}$-complete. A straightfor- 
ward application of Levi's Enlargement Lemma [18] (see also [5]) turns such an arrangement with $n$ pseudolines into a pseudolinear drawing of $K_{2 n}$ that is not stretchable. Hence, unless $\mathrm{P}=\mathrm{NP}=\exists \mathbb{R}$, there are infinitely many non-stretchable f-convex drawings of $K_{n}$.

\section{Convex drawings}

In this section we introduce the basics of convexity. We already mentioned in the introduction that the two drawings $\widetilde{\mathbb{K}}_{5}^{3}$ and $\widetilde{\mathbb{K}}_{5}^{5}$ of $K_{5}$ in Figure 1 are not convex. In fact, their absence characterizes convexity. We first prove some intermediate results that make this completely clear. Our first observation is immediate from the definition of convex side and is surprisingly useful.

Observation 2.1. If $J$ is such that $D[J]$ is a crossing $K_{4}$, and $T$ is a 3 -cycle in $J$, then the side of $D[T]$ containing the fourth vertex in $J$ is not convex.

We had some difficulty deciding on the right definition of convexity. At the level of individual 3-cycles, the definition given in the introduction makes more sense. At the level of a drawing being convex, there is a simpler one, as shown in the next lemma and, more particularly, its Corollary 2.4: we only need to test single points in the closed disc $\Delta$ and how they connect to the three corners.

Definition 2.2. Let $D$ be a drawing of $K_{n}$, let $T$ be a 3-cycle in $K_{n}$, and let $\Delta$ be a closed disc bounded by $D[T]$. Then $\Delta$ has the Four Point Property if, for every vertex $v$ of $K_{n}$ not in $T$ such that $D[v] \in \Delta, D[T+v]$ is a non-crossing $K_{4}$.

Lemma 2.3. Let $D$ be a drawing of $K_{5}$ such that the side $\Delta$ of a 3-cycle $T$ has the Four Point Property. Suppose $u$ and $v$ are vertices of $K_{5}$ such that $D[u], D[v] \in \Delta$. If $D[u v]$ is not contained in $\Delta$, then there is a vertex $b$ of $T$ such that neither side of the 3-cycle induced by $u, v$, and $b$ satisfies the Four Point Property; in particular, neither side is convex.

Proof. Since $\Delta_{T}$ has the Four Point Property, neither $u$ nor $v$ is in $T$. Because $D[u]$ and $D[v]$ are both on the same side of $D[T], D[u v]$ crosses $D[T]$ an even number of times. However, $D[u v]$ crosses each of the three sides of $D[T]$ at most once, so $D[u v]$ crosses $D[T]$ at most three times. Thus, $D[u v]$ crosses $D[T]$ either 0 or 2 times.

As $D[u v]$ is not contained in $\Delta_{T}, D[u v]$ crosses $D[T]$ a positive number of times. We conclude they cross exactly twice. Label the vertices of $T$ as $a, b$, and $c$ so that $D[u v]$ crosses both $D[a b]$ and $D[a c]$.

Since $D[T+u]$ is a non-crossing $K_{4}$, the three edges of $T+u$ incident with $u$ partition $\Delta_{T}$ into three faces, each incident with a different two of $a, b$, and $c$. Because $D[u v]$ crosses $D[a b]$ and $D[a c]$, but not any of the three edges of $T+u$ incident with $u, v$ must be in one of the faces of $D[T+u]$ incident with $a$. We choose the labelling so that $v$ is in the face of $D[T+u]$ incident with both $a$ and $c$.

The Four Point Property implies $D[v b]$ is contained in $\Delta_{T}$. It must cross either $D[u a]$ or $D[u c]$. To show that it crosses $D[u c]$, we assume by way of contradiction that it crosses $D[u a]$. Let $\times$ be the point where $D[a b]$ crosses $D[u v]$. Then $D[v b]$ must exit the region incident with $a, u$, and $\times$, but it cannot cross either $D[a b]$ or $D[u v]$, and it cannot cross $D[a u]$ a second time. This contradiction shows $D[v b]$ crosses $D[u c]$.

Therefore $D[T+\{u, v\}]$ is isomorphic to $\widetilde{\mathbb{K}}_{5}^{3}$, with $u$ and $v$ being the upper left and upper right vertices, respectively, and $a$ the peak in Figure 1. Letting $b$ and $c$ be the lower 
left and lower right vertices, respectively, the 3-cycles $u v b$ and $u v c$ have no convex side.

Obviously, if $\Delta$ is a convex side of $D[T]$, then $\Delta$ has the Four Point Property. The following converse is an immediate consequence of Lemma 2.3.

Corollary 2.4. Let $D$ be a drawing of $K_{n}$ and, for each 3-cycle $T$ in $K_{n}$, let $\Delta_{T}$ be a closed disc bounded by $D[T]$. Suppose, for each $T, \Delta_{T}$ has the Four Point Property. Then each $\Delta_{T}$ is convex; in particular, $D$ is convex.

Our next two corollaries yield additional characterizations of convexity.

Corollary 2.5. Let $D$ be a drawing of $K_{n}$.

(a) Then $D$ is not convex if and only if there exists a 3-cycle $T$ of $K_{n}$ and vertices $u$, $w$ of $K_{n}$, one in the interior of each side of $D[T]$, such that both $D[T+u]$ and $D[T+w]$ are crossing $K_{4}$ 's.

(b) If $D$ is convex and $T$ is a 3-cycle in $K_{n}$, then a side $\Delta$ of $D[T]$ is convex if and only if it satisfies the Four Point Property.

Proof. For Item (a), Observation 2.1 shows that if $D$ is convex, then no such 3 -cycle can exist. Conversely, Corollary 2.4 implies that some 3-cycle $T$ of $K_{n}$ does not have a side that satisfies the Four Point Property. This implies that, for each side $\Delta$ of $D[T]$, there is a vertex $v_{\Delta}$ such that $D\left[T+v_{\Delta}\right]$ is a crossing $K_{4}$, as required.

The proof of Item (b) is direct from the definition of convex side and Lemma 2.3.

We came to the concept of convexity by considering drawings of $K_{n}$ without the two drawings $\widetilde{\mathbb{K}}_{5}^{3}$ and $\widetilde{\mathbb{K}}_{5}^{5}$ (see Figure 1) of $K_{5}$ for reasons that have been subsumed by some of the developments described in this article. Since the remaining drawings of $K_{5}$ are rectilinear, we think of such drawings of $K_{n}$ as locally rectilinear. Our next result is the surprising equivalence with convexity and this led us to consider convexity and its strengthenings to h- and f-convex.

Theorem 2.6. A drawing $D$ of $K_{n}$ is convex if and only if, for every subgraph $J$ of $K_{n}$ isomorphic to $K_{5}, D[J]$ is not isomorphic to either $\widetilde{\mathbb{K}}_{5}^{3}$ or $\widetilde{\mathbb{K}}_{5}^{5}$.

Proof. In the drawing of $\widetilde{\mathbb{K}}_{5}^{3}$ in Figure 1, we see that a 3 -cycle consisting of one of the edges that is not a straight segment together with the longer horizontal edge has no convex side. In the drawing of $\widetilde{\mathbb{K}}_{5}^{5}$, there are two 3 -cycles that have the "interior vertex" in their interiors. Neither of these 3 -cycles is convex. Thus, these two drawings of $K_{5}$ cannot occur in a convex drawing of $K_{n}$.

Conversely, in a rectilinear drawing of $K_{5}$, the bounded side of each 3-cycle has the Four Point Property. Thus, Corollary 2.4 shows a rectilinear drawing of $K_{5}$ is convex. On the other hand, Corollary 2.5 shows every non-convex drawing of $K_{n}$ contains a nonconvex drawing of $K_{5}$. Such a drawing is either $\widetilde{\mathbb{K}}_{5}^{3}$ or $\widetilde{\mathbb{K}}_{5}^{5}$.

Theorem 2.6 has the following simple corollary. (To help with phrasing, we refer to an isomorph $J$ of $K_{r}$ to mean $J$ is a specific one of the complete subgraphs of $K_{n}$ having $r$ vertices.) 
Corollary 2.7. Let $D$ be a drawing of $K_{n}$. Suppose, for every isomorph $J$ of $K_{5}$, there is, for some $m \leq 12$, an isomorph $L$ of $K_{m}$ containing $J$ such that $D[L]$ is an optimal drawing of $K_{m}$. Then $D$ is convex.

Proof. Aichholzer [2] has verified that $D[L]$ is convex, so $D[J]$ is a convex $K_{5}$.

As illustration of the utility of Corollary 2.7, Ábrego et al. [1] exhibit, for odd $n$, a drawing $N_{n, n, 1}$ of $K_{2 n+1}$ having $H(2 n+1)$ crossings, with every edge involved in at least one crossing. We argue here that it is convex; furthermore, we believe that analogous arguments apply to any of the known examples of drawings of $K_{n}$ having $H(n)$ crossings.

We start with the tin can drawing $T C_{2 n}$ of $K_{2 n}\left(T C_{8}\right.$ is illustrated in Figure 1; in this figure, the labelling described below is counterclockwise). This has concentric regular $n$-gons, the outside one labelled $u_{0}, \ldots, u_{n-1}$ and the inside one labelled $v_{0}, \ldots, v_{n-1}$. For each $i, u_{i}$ and $v_{i}$ are roughly "antipodal", so $u_{i}$ is closest to $v_{i+\lfloor n / 2\rfloor}$. We use the non-self-crossing perfect matching consisting of the edges $u_{i} v_{i+\lfloor n / 2\rfloor}$ to work from (the indices are always read modulo $n$ ). We join $u_{i}$ to $v_{i+\lfloor n / 2\rfloor+1}, v_{i+\lfloor n / 2\rfloor+2}, \ldots, v_{i-1}$ in one direction from $u_{i} v_{i+\lfloor n / 2\rfloor}$ around the cylinder, and to $v_{i+\lfloor n / 2\rfloor-1}, v_{i+\lfloor n / 2\rfloor-2}, \ldots, v_{i+1}, v_{i}$ in the other direction. The two sides are as equal as possible. Thus, the rotation at $u_{i}$ is

$$
u_{i+1}, u_{i+2}, \ldots, u_{i-1}, v_{i}, v_{i+1}, \ldots v_{i-1} .
$$

We mentioned in the introduction that this is homeomorphic to a spherical drawing and therefore convex. However, an earlier referee requested more detail for proofs of convexity, so we provide the argument for $T C_{2 n}$ and adapt it to prove the convexity of $N_{n, n, 1}$.

To argue that $T C_{2 n}$ is convex, let $V$ be any set of five vertices in $T C_{2 n}$. These vertices are covered by at most five edges of the matching $M$ consisting of the antipodal edges $u_{i} v_{i}$. Add one or two more edges from $M$ as needed to get up to five edges, and use the induced drawing on these 10 vertices. This is $T C_{10}$ because the induced rotations of the edges at the 10 vertices satisfy the rotation described in the preceding paragraph. This is an optimal drawing of $K_{10}$, so Corollary 2.7 shows $T C_{2 n}$ is convex.

To get the Ábrego et al. drawing $N_{n, n, 1}$, add a vertex $w$ to $T C_{2 n}$ near the centre of the concentric $n$-gons. This is joined to the $2 n$ vertices by straight line segments, creating the drawing $T C_{2 n}^{+}$. The drawing $N_{n, n, 1}$ is completed by redrawing the edges $u_{i} v_{i}$ of $T C_{2 n}^{+}$as illustrated in Figure 2; these are the green edges in their Figure 4. Their labelling and ours coincide except that our indices on $u_{i}$ and $v_{i}$ are one less than theirs.

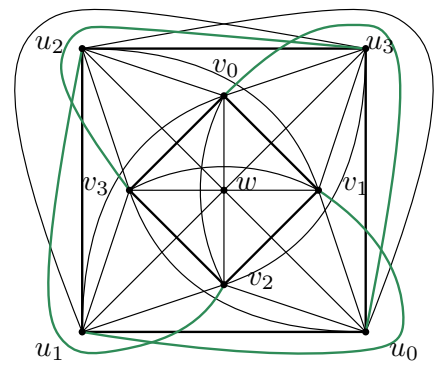

Figure 2: $N_{4,4,1}$.

Many of the $K_{5}$ 's in $N_{n, n, 1}$ are exactly the same in $T C_{2 n}^{+}$; all of these are convex. This is even true for a $K_{5}$ that contains just one green edge, say $u_{0} v_{0}$ and no vertex from 
$u_{1}, u_{2}, \ldots, u_{\lfloor n / 2\rfloor}$ : the drawings of this $K_{5}$ in $N_{n, n, 1}$ and $T C_{2 n}^{+}$are isomorphic. This is helpful in dealing with the case there is only one green edge: we may assume such a $K_{5}$ also has at least one of the $u_{j}$ listed in the preceding paragraph.

In case of a single green edge and the green edge is not involved in a crossing, we make use of the following simple observation.

Observation 2.8. Let $D$ be a convex drawing of $K_{5}$ and let $D^{\prime}$ be another (simple) drawing of $K_{5}$ obtained from $D$ by rerouting a single edge e. If $e$ is uncrossed in $D^{\prime}$, then $D^{\prime}$ is convex.

Proof. If $e$ is uncrossed in $D$, then $D$ and $D^{\prime}$ are homeomorphic drawings and the result is trivial. Otherwise, the edge $e$ is crossed in $D$, so $\operatorname{cr}\left(D^{\prime}\right)<\operatorname{cr}(D)$. These numbers are both odd (Kleitman [17]) and at most 5 . Since the spherical drawing of $K_{5}$ with one crossing is convex, the only non-trivial case is $D$ is the natural drawing of $K_{5}$.

In this case, $e$ is one of the crossed edges; these are all the same. Only the face of $G-e$ bounded by the 5 -cycle is incident with both ends of $e$. Thus, $D^{\prime}$ is a homeomorph of the rectilinear drawing whose outer face is bounded by a 4-cycle.

Next, consider a $K_{5}$ having a unique green edge crossed in the $K_{5}$. With 4 vertices determined, if the fifth vertex is $w$ or $v_{j}$, then the $K_{5}$ is optimal and hence convex. Otherwise, there are four " $u$ vertices" and it is easy to see that it is one of the rectilinear $K_{5}$ 's.

Lastly, there may be two green edges. Except for one exceptional case when $n$ is even, these edges must cross. Moreover, they give us four of the vertices of the $K_{5}$ and it is not difficult to check the different possible locations for the remaining vertex.

The following definition and corollary to Lemma 2.3 will be used in the next section for the structural description of a convex drawing.

Definition 2.9. Let $D$ be a drawing of $K_{n}$, let $u$ be a vertex of $K_{n}$, and let $J$ be a complete subgraph of $K_{n}-u$. If $D[J]$ is natural and $D[u]$ is in the face of $D[J]$ bounded by a $|V(J)|$-cycle, then $u$ is planarly joined to $J$ if no edge from $D[u]$ to $D[J]$ crosses any edge of $J$.

Corollary 2.10. Let $D$ be a convex drawing of $K_{5}$ with vertices $u, v$ such that $D-\{u, v\}$ is the 3-cycle T. If $D[u]$ and $D[v]$ are in the same face of $D[T]$ and $u$ and $v$ are both planarly joined to $T$, then $D[u v]$ is in the same face of $D[T]$ as $D[u]$ and $D[v]$.

A further perusal of the five drawings of $K_{5}$ shows that the following further refinement of forbidden substructures is possible. This configuration was mentioned at Crossing Number Workshop 2015 (Rio de Janeiro) in the context of being one forbidden configuration for a drawing of an arbitrary graph to be pseudolinear. The proof is quite straightforward.

Lemma 2.11. Let $D$ be a drawing of $K_{n}$. Then $D$ is convex if and only if, for every path $P$ of length $4, D[P]$ is not isomorphic to $\widetilde{\mathbb{P}}_{4}$.

We will use the following observation in Section 5. Its proof, left to the reader, is a good exercise in using the fact that no two closed edges can have two points in common.

Observation 2.12. Let $D$ be a drawing of $K_{5}$ in which some 3 -cycle is crossed three times by a single edge. Then $D$ is $\widetilde{\mathbb{K}}_{5}^{5}$ (as in Figure 1). 


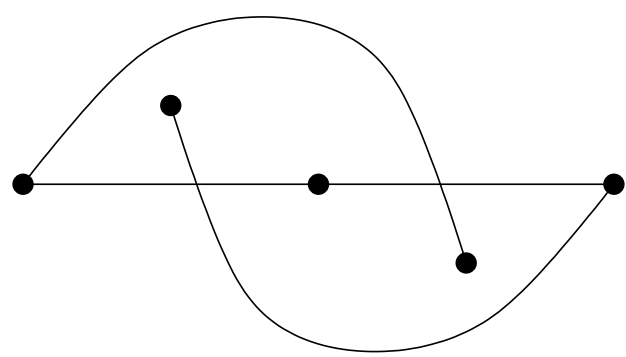

Figure 3: The drawing $\widetilde{\mathbb{P}}_{4}$.

\section{Convexity and natural drawings of $K_{n}$}

We recall from Section 1 that a natural drawing of $K_{n}$ is a drawing in which an $n$-cycle bounds a face $\Gamma$. It is easy to see that, in any natural drawing of $K_{n}$, every 3-cycle $T$ has a side $\Delta_{T}$ that is disjoint from $\Gamma$ and there is no vertex of $K_{n}$ in the interior of $\Delta_{T}$. Thus, $\Gamma$ and the $\Delta_{T}$ show that a natural drawing of $K_{n}$ is f-convex.

In this section, we show that if $D$ is a convex drawing of $K_{n}$ with the maximum number $\left(\begin{array}{l}n \\ 4\end{array}\right)$ of crossings, then $D$ is a natural drawing of $K_{n}$. This leads us to a structure theorem for convex drawings of $K_{n}$ whose central piece is, for some $r \geq 4$, a natural drawing of $K_{r}$. It also leads to the Erdős-Szekeres Theorem for convex drawings: for every $r \geq 5$, if $n$ is sufficiently large, then every convex drawing of $K_{n}$ contains a natural $K_{r}$.

Lemma 3.1. Let $D$ be a drawing of $K_{n}$. Then $D$ is a convex drawing of $K_{n}$ with $\left(\begin{array}{l}n \\ 4\end{array}\right)$ crossings if and only if $D$ is a natural drawing of $K_{n}$.

Proof. One direction is trivial; for the other, let $D$ be a convex drawing of $K_{n}$ with $\left(\begin{array}{l}n \\ 4\end{array}\right)$ crossings; all $K_{4}$ 's are crossing in $D$. We proceed by induction on $n$, the cases $n \leq 5$ being trivial. Let $v_{0}$ be any vertex of $K_{n}$ and let $H$ be the Hamilton cycle of $K_{n}-v_{0}$ such that $D[H]$ bounds a face $F$ of $D\left[K_{n}-v_{0}\right]$.

Claim 3.2. If $T$ is a 3 -cycle in $K_{n}$, then one side of $D[T]$ has no vertex drawn in its interior.

Proof. Otherwise, the convex side $\Delta$ of $D[T]$ has a vertex $v$ with $D[v]$ in the interior of $\Delta$. This yields the contradiction that $D[T+v]$ is a non-crossing $K_{4}$.

In particular, Claim 3.2 applied to all the 3-cycles in $K_{n}-v_{0}$ shows $v_{0}$ is in $F$.

Claim 3.3. Suppose $x, y, z$ are distinct vertices of $K_{n}-v_{0}$ such that $y z \in E(H)$ and the edge $D\left[v_{0} x\right]$ crosses the edge $D[y z]$. Then, for any vertex $w$ of $K_{n}-\left\{v_{0}, y, z\right\}$ :

(a) $D\left[v_{0} w\right]$ crosses $D[y z]$; and

(b) $y z$ is the only edge of $H$ crossed by $v_{0} w$.

Proof. For (a), if $D\left[v_{0} w\right]$ does not cross $D[y z]$, then the 3-cycle $D\left[v_{0} w x\right]$ has $D[y]$ and $D[z]$ on different sides, contradicting Claim 3.2.

For (b), in traversing $v_{0} w$, let $a b$ be the first edge of $K_{n}-v_{0}$ such that $D\left[v_{0} w\right]$ crosses $D[a b]$. Then $a b$ is in $H$ and $v_{0} w$ does not cross either $a w$ or $b w$. That is, the portion of $D\left[v_{0} w\right]$ from the crossing with $D[a b]$ to $D[w]$ is contained inside the 3 -cycle $D[a b w]$. 
Since every $K_{4}$ is crossing, $v_{0}$ is not planarly joined to $D-v_{0}$. Therefore, there is an edge $v_{0} x$ that crosses an edge $y z$ of $H$. Claim 3.3(a) shows that, for any vertex $w$ of $D-\left\{v_{0}, y, z\right\}, v_{0} w$ also crosses $y z$.

If $v_{0} y$ crosses some edge $y^{\prime} z^{\prime}$ of $H$, then Claim 3.3(a) shows that, for any vertex $w$ of $D-\left\{v_{0}, y^{\prime}, z^{\prime}\right\}$ also crosses $y^{\prime} z^{\prime}$. Note that $y \notin\left\{y^{\prime}, z^{\prime}\right\}$. Since $n \geq 6$, there is a $w_{0}$ different from all of $v_{0}, y, z, y^{\prime}, z^{\prime}$, so $v_{0} w_{0}$ crosses both $y z$ and $y^{\prime} z^{\prime}$, contradicting Claim 3.3(b).

Replacing the edge $y z$ of $H$ with the path $y, v_{0}, z$ yields a Hamilton cycle in $D$ that proves $D$ is a natural drawing.

The convex version of the Erdős-Szekeres Theorem is an immediate consequence of the main result of Pach et al. [23]. Their bounds are better than those coming from our Ramsey argument. Lemma 3.1 and Ramsey's Theorem also easily prove the convex version of the Erdős-Szekeres Theorem. We suppose $r \geq 5$ is an integer and choose $n$ large enough so that some subset of $V\left(K_{n}\right)$ of size $r$ is such that every $K_{4}$ in the $K_{r}$ is crossing. (For $r \geq 5$, they cannot all be non-crossing.) If the drawing $D$ of $K_{n}$ is convex, Lemma 3.1 implies $D\left[K_{r}\right]$ is natural. We state the theorem here for reference.

Theorem 3.4. Let $r \geq 5$ be an integer. Then there is an integer $N=N(r)$ such that, if $n \geq N$ and $D$ is a convex drawing of $K_{n}$, then there is a subgraph $J$ of $K_{n}$ isomorphic to $K_{r}$ such that $D[J]$ is a natural $K_{r}$.

The remainder of this section is devoted to a structure theorem for convex drawings. Let $D$ be a convex drawing of $K_{n}$ and, for some $r \geq 4$, let $J$ be a $K_{r}$ in $K_{n}$ such that $D[J]$ is natural. We set $C_{J}$ to be the facial $r$-cycle in $D[J]$. We refer to the face of $D[J]$ bounded by $C_{J}$ as the outside of $J$ and the other side of $C_{J}$ as the inside of $J$.

The proof uses the following elementary observations that are somewhat interesting and otherwise useful in their own right.

Lemma 3.5. Let $D$ be a convex drawing of $K_{n}$ and, for some $r \geq 4$, let $J$ be a $K_{r}$ such that $D[J]$ is natural, with facial $r$-cycle $C_{J}$.

(a) If $u$ is inside $J$, then, for each $v \in V(J), D[u v]$ is inside $J$.

(b) If $u$ and $v$ are both inside $J$, then $D[u v]$ is inside $J$.

(c) If $u$ and $v$ are both outside $J$ and planarly joined to $J$, then $D[u v]$ is contained in the outside of $J$.

(d) Let $u$ be outside of $J$ and suppose there is a vertex $v$ of $J$ such that $D[u v]$ crosses $C_{J}$. Then $D[u v]$ crosses $C_{J}$ exactly once.

(e) Suppose $u$ is outside of J but, for vertices $v$ and $w$ of J, $D[u v]$ and $D[u w]$ both cross $C_{J}$. Let $e$ and $f$ be the edges of $C_{J}$ crossed by $D[u v]$ and $D[u w]$. Then $v$ and $w$ are in the same component of $C_{J}-\{e, f\}$.

(f) Suppose $u$ is outside of $J, v$ is a vertex of $J$, and $D[u v]$ crosses $C_{J}$ on the edge ab. Then $D[u a]$ and $D[u b]$ are contained in the outside of $J$.

Proof. We start with (a). If we consider the edges of $J$ incident with $v$, they partition the inside of $J$ into discs bounded by 3-cycles. As $|V(J)| \geq 4$, the disc containing $u$ is the convex side of its bounding 3 -cycle. Thus, $D[u v]$ is inside this disc and so is inside $J$. 
For (b), we present an argument suggested by Kasper Szabo Lyngsie that simplifies our original. There is an edge $x y$ in $C_{J}$ such that $v$ is in the side $\Delta$ of $D[u x y]$ that has no vertices of $J-\{x, y\}$. If there is an edge of $J$ incident with either $x$ or $y$ that crosses the 3 -cycle $u x y$, then $v$ is in the crossing side of a natural $K_{4}$ containing $u, x$, and $y$. In this case, $\Delta$ is the convex side of $u x y$, so $D[u v]$ is inside $\Delta$.

In the other case, let $x^{\prime}$ and $y^{\prime}$ be the neighbours of $x$ and $y$, respectively, in $C_{J}-x y$. Then $\Delta$ is contained in the convex side $\Delta^{\prime}$ of the 3 -cycle $x^{\prime} x y$, and again $D[u v]$ is contained in $\Delta^{\prime}$ and consequently inside $J$. (We remark that, in fact, $D[u v]$ is contained inside $\Delta$, but $v x$ or $v y$ might cross $u x y$, so $\Delta$ need not be the convex side of $u x y$.)

Moving on to (c), let $x, y, z$ be any three vertices of $J$ and let $L$ be the $K_{5}$ induced by $u, v, x, y, z$. Then $D[L]$ is a convex drawing of $K_{5}$. Let $T$ be the 3 -cycle $(x, y, z)$. The assumption that $u$ and $v$ are planarly joined to $T$ in $D$ shows that the side $\Delta_{T}$ of $T$ that contains $u$ and $v$ satisfies the Four Point Property in $D[L]$.

Corollary 2.10 implies that $D[u v]$ is contained in $\Delta_{T}$. This is true for every three vertices of $J$, so $D[u v]$ is contained in the intersection of all the $\Delta_{T}$ 's; this is precisely the closure of the face of $D[J]$ containing $D[u]$ and $D[v]$, as required.

In the proof of (d), we suppose the first crossing of $u v$ is with the edge $x y$ of $C_{J}$. The 3 -cycle $x y v$ is inside $J$ and, by the definition of drawing, $u v$ cannot cross $x y v$ a second time.

Turning to (e), we suppose that $v$ and $w$ are in different components of $C_{J}-\{e, f\}$ and that $u v$ crosses $e$, while $u w$ crosses $f$. Let $x$ be the end of $e$ in the component of $C_{J}-\{e, f\}$ containing $v$ and let $y$ be the end of $f$ in the component of $C_{J}-\{e, f\}$ containing $w$. By the definition of drawing, $x \neq v$ and $y \neq w$.

The edge $x w$ crosses $u v$ and the edge $y v$ crosses $u w$. Moreover, $x$ and $y$ are on different sides of the 3-cycle $u v w$, so $u v w$ has no convex side, a contradiction.

For (f), it suffices by symmetry to show $D[u a]$ is outside $J$. In the alternative, $u a$ crosses $C_{J}$. Since it cannot cross $u v$ by goodness, it must cross the $a v$-subpath of $C_{J}-a b$. But now $u a$ and $u v$ violate (e).

We now turn to the basic ingredient in the structure theorem. Let $D$ be a convex drawing of $K_{n}$ and, for some $r \geq 4$, let $J$ be a $K_{r}$ such that $D[J]$ is natural. The $J$-induced drawing $\bar{J}$ consists of the subdrawing induced by $D[J]$ and all vertices inside of $J$. The following is the main point in the proof of the structure theorem.

Lemma 3.6. Let $D$ be a convex drawing of $K_{n}$ and, for some $r \geq 4$, let $J$ be a $K_{r}$ such that $D[J]$ is natural. If there is a vertex $u$ outside $J$ and a vertex $v$ of $J$ such that $D[u v]$ crosses $C_{J}$, then there is, for some $s \geq 4$, a $K_{s}$-subgraph $J^{\prime}$ including $u$ such that $D\left[J^{\prime}\right]$ is natural and $\bar{J} \subset \bar{J}^{\prime}$.

Proof. Let $a b$ be the edge of $C_{J}$ crossed by $u v$. Lemma 3.5(f) implies that $D[u a]$ and $D[u b]$ are contained in the outside of $J$. It follows that, in the $a v$-subpath of $C_{J}-a b$, there is a vertex $w_{a}$ nearest $v$ such that $D\left[u w_{a}\right]$ is contained in the outside of $J$. Likewise, there is a nearest such vertex $w_{b}$ in the $b v$-subpath.

For any internal vertex $x$ in the $w_{a} w_{b}$-subpath $P$ of $C_{J}-a b$, the edge $u x$ must cross $C_{J}$; Lemma 3.5(d) and (f) imply $u x$ must cross $w_{a} w_{b}$. It follows that $u x$ does not cross $P$. Thus, the cycle consisting of $u$, together with $P$, makes the facial cycle for a natural $K_{s}$ $(s=1+|V(P)| \geq 4)$ and all the points of $\bar{J}$ are on or inside this $K_{s}$.

Our structure theorem is an immediate consequence of Lemmas 3.5(c) and 3.6. 
Theorem 3.7 (Structure Theorem). Let $n \geq 5$ and let $D$ be a convex drawing of $K_{n}$. Then, for some $r \geq 4$, there is a $K_{r}$-subgraph $J$ such that $D[J]$ is natural, every vertex outside of $J$ is planarly joined to $J$, and any two vertices outside $J$ are joined outside $J$.

As a consequence of the Structure Theorem, we have the following straightforward, though not trivial, observation. As it is not of immediate interest, we give only a proof sketch.

Theorem 3.8. Let $n \geq 5$ and let $D$ be a drawing of $K_{n}$. Suppose that, for every subgraph $J$ of $K_{n}$ that is isomorphic to a $K_{4}$ and $D[J]$ has a crossing, there are no vertices of $K_{n}$ inside $D[J]$. Then $D\left[K_{n}\right]$ is convex and either:

1. a natural $K_{n}$; or

2. a natural $K_{n-1}$ with one vertex outside that is planarly joined to the $K_{n-1}$; or

3. the unique drawing of $K_{6}$ with three crossings.

Proof sketch. The hypothesis on the crossing $K_{4}$ 's implies the drawing is convex: in both $\widetilde{\mathbb{K}}_{5}^{3}$ and $\widetilde{\mathbb{K}}_{5}^{5}$, there is a crossing $K_{4}$ with a vertex inside the $K_{4}$.

Apply the Structure Theorem 3.7 to $D$ to get a subgraph $J$ of $K_{n}$ such that $D[J]$ is a natural $K_{r}$, with $r \geq 4$ and every other vertex of $K_{n}$ is either inside $D[J]$ or is outside $J$ and planarly joined to $J$.

Any vertex inside $D[J]$ is in a face that is incident with a crossing of some crossing $K_{4}$ involving four vertices in $J$. Since this is forbidden, there is no vertex inside $D[J]$.

If there are three vertices of $K_{n}$ outside $D[J]$, then there is a crossing $K_{4}$ with a vertex inside.

If there are two vertices $u, v$ of $K_{n}$ outside $D[J]$ and some edge from $u$ to $J$ crosses two edges from $v$ to $J$, then there is a crossing $K_{4}$ with a vertex inside. In particular, if $r \geq 5$, then there is at most one vertex outside $J$.

The remaining case is $r=4$ and no $u J$-edge crosses two $v J$-edges and no $v J$-edge crosses two $u J$-edges. This is the unique drawing of $K_{6}$ with three crossings.

In general, if, in a convex drawing of $K_{n}$, we bound by a non-negative integer $p$ the number of vertices allowed inside any natural $K_{4}$, there is a theorem in the spirit of Theorem 3.8. There are more special cases with $n$ small, but if $n$ is large enough (on the order of $3 p$ ), the structure is: a natural $K_{r}$, with $r$ at least roughly $p / 3$, and at most one of the remaining points is outside the natural $K_{r}$.

\section{4 h-convex drawings}

In this section, we investigate h-convex drawings. Our main result is a characterization of h-convex drawings; this immediately yields a polynomial time algorithm for determining if a drawing is h-convex.

Consider the drawing $\mathbb{K}_{6}^{11}$. It is convex, but not h-convex. To see that it is convex, it suffices to check the six $K_{5}$ 's and observe that none of them is either $\widetilde{\mathbb{K}}_{5}^{3}$ or $\widetilde{\mathbb{K}}_{5}^{5}$. To see that it is not h-convex, consider the dashed $K_{4}$ (including the thick edge) highlighted in Figure 1. For this $K_{4}$, either of the 3 -cycles $T$ containing the thick edge has its bounded (in the figure) side convex, while its unbounded side is not convex. A similar statement holds 
for the a 3-cycle in the dotted $K_{4}$ that contains the thick edge (bounded and unbounded sides reversing roles). These 3 -cycles show that $D$ is not h-convex.

Definition 4.1. Let $D$ be a drawing of $K_{n}$ and let $J$ and $J^{\prime}$ be distinct $K_{4}$ 's in $D$ such that both $D[J]$ and $D\left[J^{\prime}\right]$ are crossing $K_{4}$ 's. For 3 -cycles $T$ and $T^{\prime}$ in $J$ and $J^{\prime}$, respectively, let $\Delta_{T}$ and $\Delta_{T^{\prime}}$ be the sides of $T$ and $T^{\prime}$, respectively, not containing the fourth vertex of $J$ and $J^{\prime}$, respectively. Then $J$ and $J^{\prime}$ are inverted $K_{4}$ 's in $D$ if there are 3-cycles $T$ in $J$ and $T^{\prime}$ in $J^{\prime}$ such that $D[T] \subseteq \Delta_{T^{\prime}}$ but $\Delta_{T} \nsubseteq \Delta_{T^{\prime}}$.

The following observation is immediate from the definition.

Observation 4.2. Let $J$ and $J^{\prime}$ be inverted $K_{4}$ 's in a drawing $D$ of $K_{n}$ and let $T$ and $T^{\prime}$ be 3-cycles in $J$ and $J^{\prime}$, respectively. Let $\Delta_{T}$ and $\Delta_{T^{\prime}}$ be the sides of $T$ and $T^{\prime}$, respectively, not containing the fourth vertex of $J$ and $J^{\prime}$, respectively. If $D[T] \subseteq \Delta_{T^{\prime}}$ but $\Delta_{T} \nsubseteq \Delta_{T^{\prime}}$, then $D\left[T^{\prime}\right] \subseteq \Delta_{T}$ but $\Delta_{T^{\prime}} \nsubseteq \Delta_{T}$.

We are ready for our first characterization of h-convex drawings.

Lemma 4.3. Let $D$ be a convex drawing of $K_{n}$. Then $D$ is h-convex if and only if there are no inverted $K_{4}$ 's.

Proof. It is clear that if $D$ is h-convex, then there are no inverted $K_{4}$ 's.

For the converse, we shall inductively obtain a list $\mathcal{C}$ of convex sides, one for each 3 cycle of $K_{n}$. Along the way, the list $\mathcal{C}$ will have convex sides for some, but not all, of the 3 -cycles of $K_{n}$. Such a partial list is hereditary if, for any 3-cycles $T$ and $T^{\prime}$ having convex sides $\Delta_{T}$ and $\Delta_{T^{\prime}}$, respectively, in $\mathcal{C}$, if $D[T] \subseteq \Delta_{T^{\prime}}$, then $\Delta_{T} \subseteq \Delta_{T^{\prime}}$.

Our initial list $\mathcal{C}_{0}$ consists of the convex sides for every 3 -cycle that is in a crossing $K_{4}$. The assumption that there are no inverted $K_{4}$ 's immediately implies $\mathcal{C}_{0}$ is hereditary.

Let $T_{1}, \ldots, T_{r}$ be the 3 -cycles in $K_{n}$ such that, for $i=1,2, \ldots, r, T_{i}$ is not in any crossing $K_{4}$. For $j \geq 1$, suppose that $\mathcal{C}_{j-1}$ is a hereditary list of convex sides that includes $\mathcal{C}_{0}$ and a convex side for each of $T_{1}, \ldots, T_{j-1}$.

If there is a convex side $\Delta_{T} \in \mathcal{C}_{j-1}$ such that $D\left[T_{j}\right] \subseteq \Delta_{T}$, then we choose $\Delta_{T_{j}}$ so that $\Delta_{T_{j}} \subseteq \Delta_{T}$. Otherwise, we choose $\Delta_{T_{j}}$ arbitrarily from the two sides of $D\left[T_{j}\right]$. Set $\mathcal{C}_{j}=\mathcal{C}_{j-1} \cup\left\{\Delta_{T_{j}}\right\}$.

We show that $\mathcal{C}_{j}$ is hereditary. If not, then, since $\mathcal{C}_{j-1}$ is hereditary, there is a 3 cycle $T$ with a convex side $\Delta_{T} \in \mathcal{C}_{j-1}$ such that either $D[T] \subseteq \Delta_{T_{j}}$ and $\Delta_{T} \nsubseteq_{T_{j}}$ or $D\left[T_{j}\right] \subseteq \Delta_{T}$ and $\Delta_{T_{j}} \nsubseteq \Delta_{T}$. The second case implies that $D[T] \subseteq \Delta_{T_{j}}$ and $\Delta_{T} \nsubseteq \Delta_{T_{j}}$, which is the first case.

Thus, in both cases, we have that $D[T] \subseteq \Delta_{T_{j}}$ and $\Delta_{T} \nsubseteq \Delta_{T_{j}}$. By the choice of $\Delta_{T_{j}}$, there is a second already considered triangle $T^{\prime}$ such that $D\left[T^{\prime}\right]$ is contained in the other side $\bar{\Delta}_{T_{j}}$ of $D\left[T_{j}\right]$ but $\Delta_{T^{\prime}} \not \bar{\Delta}_{T_{j}}$.

Evidently, $D[T] \subseteq \Delta_{T^{\prime}}$ and $\Delta_{T} \nsubseteq \Delta_{T^{\prime}}$, yielding the contradiction that $\mathcal{C}_{j-1}$ is not hereditary.

Lemma 4.3 yields an $\mathrm{O}\left(n^{8}\right)$ algorithm for determining whether a drawing is h-convex. Also, a similar argument proves the following analogous fact for f-convexity. This is essentially the characterization of pseudolinearity due to Aichholzer et al. [4].

Theorem 4.4 ([4]). Let $D$ be a drawing of $K_{n}$. Then $D$ is $f$-convex if and only if there is a face $\Gamma$ such that, for every isomorph $J$ of $K_{4}$ for which $D[J]$ is a crossing $K_{4}, \Gamma$ is contained in the face of $D[J]$ bounded by the 4-cycle. 
There is a colourful way to understand this theorem. For each isomorph $J$ of $K_{4}$ for which $D[J]$ is a crossing $K_{4}$, let $C_{J}$ be the 4-cycle in $J$ that bounds a face of $D[J]$. Paint the side of $D\left[C_{J}\right]$ that contains the crossing of $D[J]$. If the whole sphere is painted, then $D$ is not f-convex. Otherwise, with respect to any face $F$ of $D\left[K_{n}\right]$ that is not painted, $F$ witnesses that $D$ is f-convex.

Our next result gives a surprising characterization of h-convex drawings of $K_{n}$ by a single additional forbidden configuration.

Theorem 4.5. Let $D$ be a convex drawing of $K_{n}$. Then $D$ is h-convex if and only if, for each isomorph $J$ of $K_{6}$ in $K_{n}, D[J]$ is not isomorphic to $\mathbb{K}_{6}^{11}$.

Proof. Since h-convexity is evidently inherited by induced subgraphs, no h-convex drawing of $K_{n}$ can contain $\mathbb{K}_{6}^{11}$. Conversely, suppose $D$ is not h-convex; we show $D$ contains $\mathbb{K}_{6}^{11}$.

By Lemma 4.3, there exist isomorphs $J_{1}$ and $J_{2}$ of $K_{4}$ that are inverted in $D$. For $i=1,2$, let $T_{i}$ be a 3 -cycle in $J_{i}$ with convex side $\Delta_{T_{i}}$ such that $D\left[T_{1}\right] \subseteq \Delta_{T_{2}}$ and $\Delta_{T_{1}} \not \Delta_{T_{2}}$.

Let $w$ be the vertex of $J_{1}$ not in $T_{1} ; D[w]$ is separated from $D\left[T_{2}\right]$ by $D\left[T_{1}\right]$. Let $x$ be the vertex of $T_{1}$ such that $D[w x]$ crosses $D\left[T_{1}\right]$. Complete $D[w x]$ to a simple closed curve $\gamma$ by adding a segment on the non-convex side of $D\left[T_{1}\right]$ joining $D[w]$ and $D[x]$. Clearly $\gamma$ separates the two vertices of $T_{1}-x$. Moreover, $D\left[T_{1}\right]$ and, therefore, $D[w]$ as well, are all contained in $\Delta_{2}$. Convexity implies $D\left[J_{1}\right] \subseteq \Delta_{2}$. Thus, $\gamma$ also separates one of the vertices of $T_{1}-x$ from $D\left[T_{2}\right]$; let $z$ be the one separated from $T_{2}$ by $\gamma$ and let $y$ be the other.

Since $D\left[T_{1}\right] \subseteq \Delta_{T_{2}}, D\left[T_{2}+z\right]$ is a non-crossing $K_{4}$. If any of the edges from $z$ to $T_{2}$ crosses $T_{1}$, then we have proof that the side $\Delta_{T_{1}}$ of $D\left[T_{1}\right]$ is not convex, a contradiction. Therefore, $D\left[T_{1}\right]$ is contained in a face $\Gamma$ of $D\left[T_{2}+z\right]$ that is incident with $z$. It follows that $w$ is also in $\Gamma$.

Let $a$ be the vertex of $T_{2}$ not incident with $\Gamma$. The edge $w x$ has both its ends in $\Gamma$. Since $\gamma$ separates $z$ from $T_{2}, \gamma$ must cross $z a$ and, therefore, is not contained in $\Gamma$. It follows that $\Gamma$ is not the convex side of the 3 -cycle $T_{3}$ that bounds $\Gamma$.

Evidently, $D\left[T_{3}\right] \subseteq \Delta_{T_{2}}$ and $\Delta_{T_{3}} \nsubseteq \Delta_{T_{2}}$. Corollary 2.5(b) implies that there is a vertex $v_{3}$ such that $v_{3} \in \Gamma$ and $D\left[T_{3}+v_{3}\right]$ is a crossing $K_{4}$. Because $T_{2}$ is in the isomorph $J_{2}$ of $K_{4}$, there is a vertex $v_{2}$ in $J_{2}$ that is not in $T_{2}$. Since $D\left[J_{2}\right]$ is a crossing $K_{4}, D\left[v_{2}\right] \notin \Delta_{T_{2}}$.

We now consider the isomorph of $K_{6}$ consisting of $\left(T_{2} \cup T_{3}\right)+\left\{v_{2}, v_{3}\right\}$. Because $D\left[\left(T_{2} \cup T_{3}\right)+v_{2}\right]$ is contained in $\Delta_{T_{3}}$, no edge from $v_{2}$ to a vertex in $T_{2} \cup T_{3}$ can cross $D\left[T_{3}\right]$. In particular, (recall that $a$ is the vertex of $T_{2}$ not in $T_{3}$ ) $D\left[v_{2} a\right]$ does not cross $D\left[T_{2} \cup T_{3}\right]$. Let $b$ be the vertex of $T_{2}$ such that $D\left[v_{2} b\right]$ crosses $D\left[T_{2}\right]$ and let $c$ be the third vertex of $T_{2}$.

Symmetrically, $z$ is the vertex of $T_{3}$ that is not in $T_{2}$, so $D\left[v_{3} z\right]$ does not cross $D\left[T_{2} \cup T_{3}\right]$. As both $D[z]$ and $D\left[v_{2}\right]$ are in $\Delta_{T_{3}}$, the edge $z v_{2}$ cannot cross $T_{3}$. Since $D\left[v_{2} b\right]$ crosses $D[a c]$ but not $D\left[T_{3}\right]$, it must also cross $D[a z]$. It follows that $D\left[v_{2} z\right]$ crosses only $D[a c]$.

Let $b^{\prime}$ be the one of $b$ and $c$ such that $D\left[v_{3} b^{\prime}\right]$ crosses $D\left[T_{3}\right]$ and let $c^{\prime}$ be the other. There are two cases to consider: $b=b^{\prime}$ and $c=c^{\prime}$; or $b=c^{\prime}$ and $c=b^{\prime}$. Note that, in each case, convexity and the definitions of $b$ and $b^{\prime}$ determine the routings of all the edges except $v_{2} v_{3}$ and $v_{3} a$.

Let $T_{4}$ be the 3 -cycle induced by $b, v_{2}$ and $c$. Since $D[a c]$ crosses $D\left[v_{2} b\right]$, the convex side of $D\left[T_{4}\right]$ is the side that contains $v_{3}$. Thus, $D\left[v_{2} v_{3}\right]$ must be contained in this side of 
$D\left[T_{4}\right]$. In the case $b=b^{\prime}, D\left[v_{3} b\right]$ crosses $D[c z], D\left[z v_{2}\right]$, and $D[a z]$. Thus, the only routing for $D\left[v_{2} v_{3}\right]$ is across $D[a c]$ and $D[z c]$. In the case $b=c^{\prime}$, the only routing for $D\left[v_{2} v_{3}\right]$ is across $D[a c], D[a z]$, and $D[z b]$. In both cases there is only one routing available for $D\left[v_{3} a\right]$.

To see in each case that these drawings are both $\mathbb{K}_{6}^{11}$, focus on the face-bounding 4cycles induced by $b, a^{\prime}, v_{3}, c$ and $b, a, v_{2}, c$.

The previous results were about a drawing being h-convex. The following result characterizes when a collection of sides, one for each 3-cycle of $K_{n}$, is a set of h-convex sides. Its proof is similar to the proof of Theorem 4.5.

Lemma 4.6. Let $D$ be a drawing of $K_{n}$ and, for each 3-cycle $T$ of $K_{n}$, let $\Delta_{T}$ be one of the closed discs bounded by $D[T]$. Let $\mathcal{C}$ be the set of all these $\Delta_{T}$. Then $\mathcal{C}$ is a set of $h$-convex sides if and only if both of the following hold:

(1) each $\Delta_{T}$ has the Four Point Property; and

(2) for each non-crossing $K_{4}$, at least three of the four (closed) faces of the non-crossing $K_{4}$ are in $\mathcal{C}$.

Proof. Let $\mathcal{C}$ be a set of h-convex sides. They are also convex sides, so obviously satisfy (1). If $J$ is a non-crossing $K_{4}$ and $T$ is a 3-cycle in $J$, then the side $\Delta_{T}$ of $T$ that is in $\mathcal{C}$ is either empty or contains $J$; in the latter case all the empty sides of the other 3 -cycles in $J$ are in $\mathcal{C}$ by heredity. Thus, at most one of the four 3 -cycles in $J$ has non-empty side in $\mathcal{C}$, which is (2).

Conversely, let $\mathcal{C}$ satisfy (1) and (2). Then $\mathcal{C}$ is a set of convex sides by Corollary 2.4. Now let $T, T^{\prime}$ be 3 -cycles such that $D\left[T^{\prime}\right] \subseteq \Delta_{T}$.

Suppose there is a $t \in T \backslash T^{\prime}$ such that $T^{\prime}+t$ is a crossing $K_{4}$. Then the convex side $\Delta_{T^{\prime}}$ of $T^{\prime}$ is the side that does not contain $t$. Because $D\left[T^{\prime}\right] \subseteq \Delta(T)$, this implies $\Delta_{T^{\prime}} \subseteq \Delta(T)$, as required. Thus, we may assume $T^{\prime}$ is not crossed in $D$.

Let $v$ be any vertex of $T \backslash T^{\prime}$. Then $D[T+v]$ is a non-crossing $K_{4}$. By (2), each of the three faces of $D[T+v]$ incident with $v$ is the side of the bounding 3 -cycle that is in $\mathcal{C}$.

We proceed by induction on the number of vertices of $T^{\prime}$ that are not in $T$; this number being at least 1 . Because $T^{\prime}$ is not crossed in $D$, the remaining vertices of $T^{\prime}$ are either in or incident with the same face $F$ of $T+v$. Let $T^{\prime \prime}$ be the 3-cycle bounding $F$.

The base of the induction is that $T^{\prime}-v \subseteq T$. In this case, $T^{\prime}=T^{\prime \prime}$ and $\Delta_{T^{\prime}}$ is $F$; thus, $\Delta_{T^{\prime}} \subseteq \Delta_{T}$, as required.

For the induction step, suppose $\left|T^{\prime} \backslash T\right| \geq 2$. We have already seen that the 3 -cycle $T^{\prime \prime}$ bounding $F$ has $\Delta_{T^{\prime \prime}}=F$. For this induction step, there is a vertex $w$ of $T^{\prime}$ in the interior of $F$, so the side of $\Delta_{T^{\prime \prime}}$ in $\mathcal{C}$ is not a face of $T^{\prime \prime}+w$. Now $T^{\prime} \subseteq \Delta_{T^{\prime \prime}}$ and $\left|T^{\prime} \backslash T^{\prime \prime}\right|<\left|T^{\prime} \backslash T\right|$. By induction, $\Delta_{T^{\prime}} \subseteq \Delta_{T^{\prime \prime}}$. Since $\Delta_{T^{\prime \prime}} \subseteq \Delta_{T}$, we have shown $\mathcal{C}$ is the set of h-convex sides, as required.

Although Lemma 4.6 shows that h-convexity is determined by considering all sets of four points, it is not evident that there is an $\mathrm{O}\left(n^{4}\right)$ algorithm to test whether a drawing is h-convex. Theorem 4.5 makes it clear that there is an $\mathrm{O}\left(n^{6}\right)$ algorithm to determine if a drawing of $K_{n}$ is h-convex.

It is $\mathrm{O}\left(n^{4}\right)$ to check that a drawing is convex. To see this, we use the Four Point Property (Corollary 2.4): for each 3-cycle $T$ and each vertex $v$, we determine which side of $T v$ is on and whether $T+v$ is a planar $K_{4}$. 
We conclude this section with an observation related to the Structure Theorem 3.7.

Lemma 4.7. Let $D$ be an h-convex drawing of $K_{n}$ consisting of a natural $K_{r}$ (with $r \geq 4$ ) and all other points inside the natural $K_{r}$. Then $D$ is f-convex.

Proof. Any triangle of the $K_{r}$ has its convex side determined; it is the side that avoids the face $F$ bounded by the cycle $C_{r}$. Let $T$ be any other triangle. If an edge of $K_{r}$ crosses $T$, then the convex side of $T$ must also avoid $F$. If this does not happen, then $T$ is inside a triangle of $K_{r}$ and the result follows from the hereditary property.

\section{Suboptimal drawings of $K_{n}$ having either $\widetilde{\mathbb{K}}_{5}^{3}$ or $\widetilde{\mathbb{K}}_{5}^{5}$}

In this section, we prove that a broad class of "locally determined" drawings of $K_{n}$ are suboptimal. This is the first theorem of its type. The theorem requires the presence of either $\widetilde{\mathbb{K}}_{5}^{3}$ or $\widetilde{\mathbb{K}}_{5}^{5}$ in the drawing, but, for at least one such $K_{5}$, the occurrence is restricted. This might be a first step towards showing that all optimal drawings of $K_{n}$ are convex.

This line of research was stimulated by Tilo Wiedera's computation (personal communication) showing that any drawing of $K_{9}$ that contains a $\widetilde{\mathbb{K}}_{5}^{5}$ has at least 40 crossings. This is in line with Aichholzer's later computations (see the remark following the statement of Theorem 5.1 below).

We also rethink the approach in [21] that $\operatorname{cr}\left(K_{9}\right)=36$. This was done before convexity became known to us. Using the fact that $\operatorname{cr}\left(K_{7}\right)=9$, it is easy to see that $\operatorname{cr}\left(K_{9}\right) \geq 34$. At the end of this section, we show easily by hand that there is no non-convex drawing $D$ of $K_{9}$ such that $\operatorname{cr}(D)=34$. Thus, to prove that $\operatorname{cr}\left(K_{9}\right)=36$, it suffices to consider convex drawings of $K_{9}$.

A principal result of this work is the following, which shows that if $D$ is a drawing of $K_{n}$ such that some non-convex $K_{5}$ intersects every other non-convex $K_{5}$ in at most two vertices, then $D$ is not optimal.

Theorem 5.1. Let $D$ be a drawing of $K_{n}$ such that there is an isomorph $J$ of $K_{5}$ with $D[J]$ either $\widetilde{\mathbb{K}}_{5}^{3}$ or $\widetilde{\mathbb{K}}_{5}^{5}$. Suppose, for every isomorph $H$ of $K_{7}$ in $K_{n}$ containing $J, D[J]$ is the only non-convex $K_{5}$ in $D[H]$.

1. If $J$ is $\widetilde{\mathbb{K}}_{5}^{3}$, then there is a drawing $D^{\prime}$ of $K_{n}$ such that $\operatorname{cr}\left(D^{\prime}\right) \leq \operatorname{cr}(D)-2$.

2. If $J$ is $\widetilde{\mathbb{K}}_{5}^{5}$, there is a drawing $D^{\prime}$ of $K_{n}$ such that $\operatorname{cr}\left(D^{\prime}\right) \leq \operatorname{cr}(D)-4$. If, in addition, $n$ is even, then $\operatorname{cr}\left(D^{\prime}\right) \leq \operatorname{cr}(D)-5$.

We remark that the lower bounds 2,4 , and 5 for $\operatorname{cr}(D)-\operatorname{cr}\left(D^{\prime}\right)$ exhibited in Theorem 5.1 are precisely the smallest differences found by Aichholzer (private communication) between any drawing, for $n \leq 12$, of $K_{n}$ that has either a $\widetilde{\mathbb{K}}_{5}^{3}$ or a $\widetilde{\mathbb{K}}_{5}^{5}$ and an optimal drawing of $K_{n}$.

Proof of Theorem 5.1. We use the labelling of $J$ as shown in Figure 4. We first deal with the case $J=\widetilde{\mathbb{K}}_{5}^{3}$.

(I) $J=\widetilde{\mathbb{K}}_{5}^{3}$.

Claim 5.2. There is no vertex of $D\left[K_{n}\right]$ in the side of any of the 3 -cycles $D[$ stw], $D[$ suv], and $D[t u v]$ that has no vertex of $D[J]$. 

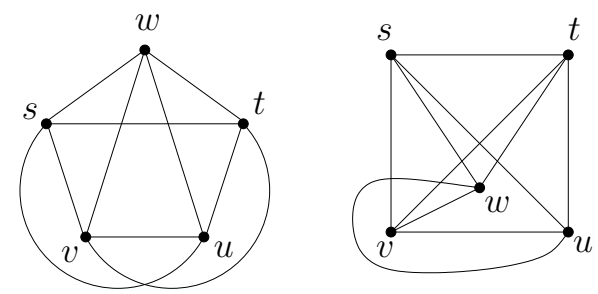

Figure 4: Labelled $\widetilde{\mathbb{K}}_{5}^{3}$ and $\widetilde{\mathbb{K}}_{5}^{5}$ for the proof of Theorem 5.1.

Proof of Claim 5.2. We start with $D[s t w]$. Similar arguments apply to $D[s u v]$. Finally, symmetry shows that $D[t u v]$ also does not have a vertex on the side empty in $D[J]$.

Suppose to the contrary that there is a vertex $x$ of $K_{n}$ such that $D[x]$ is in the side of $D[s t w]$ that is empty in $D[J]$. By hypothesis, the $K_{5}$ consisting of $J-w$ plus $x$ is convex in $D$. Since $D[x]$ is incident with a face of $D[J-w]$ that is incident with the crossing of $D[J-w]$, Observation 2.1 and convexity imply $D[x u]$ does not cross the 4-cycle $D[$ stuv $]$.

Likewise, the $K_{5}$ consisting of $J-v$ together with $x$ is convex in $D$. Again, $D[x]$ is in a face of $D[J-v]$ incident with a crossing, so $D[x u]$ does not cross the 4-cycle $D[w t u s]$. However, $D[x]$ and $D[u]$ are in different faces of $D[s t u v] \cup D[w t u s]$, so $D[x u]$ must cross at least one of the two 4-cycles.

The same deletions show that any vertex in the empty side of $D[s u v]$ cannot connect to $t$.

There are two remaining regions of interest. Let $\times$ be the crossing of $s u$ with $t v$. Let $R_{1}$ be the region bounded by $D[w t \times s w]$ that does not contain $D[u]$ and $D[v] ; R_{2}$ is the region bounded by $D[s t u v]$ that does not contain $D[w]$.

The following observations follow immediately from the convexity of the corresponding $K_{5}$ and the knowledge of the crossings.

(Obs. 1) If $x \in R_{1}$, then $J-w+x$ is convex, so the routings of $x$ to $s, t, u, v$ are determined.

(Obs. 2) If $y \in R_{2}$, then $J-u+y$ and $J-v+y$ are convex, so the routings of $y$ to $J$ are determined. $\square$

These observations yield all of (1) - (5) in the following assertions except for (3).

Claim 5.3. If $D[x] \in R_{1}$ and $D[y] \in R_{2}$, then:

(1) $D[x u]$ crosses $D[J]$ only on $D[t v]$ and $D[x v]$ crosses $D[J]$ only on $D[s u]$;

(2) $D[x s]$ and $D[x t]$ do not cross $D[J]$;

(3) $D[x w]$ either does not cross $D[J]$, or crosses $D[s t]$ and at least one of $D[s u]$ and $D[t v]$

(4) $D[y s], D[y t], D[y u]$, and $D[y v]$ cross $D[J]$ at most in either $D[u w]$ or $D[v w]$ (or both);

(5) $D[y w]$ crosses only $D[s t]$. 
Moreover, if $z z^{\prime}$ is an edge of $G$ with neither $z$ nor $z^{\prime}$ in $J$ and $T$ is one of the 3-cycles stw, suv, and tuv, then either $D\left[z z^{\prime}\right]$ does not cross $D[T]$ or it crosses the one of $D[s t]$, $D[s u]$, and $D[t v]$ that is in $D[T]$.

Proof of Claim 5.3. For $x w$, the following argument is due to Matthew Sullivan, simplifying our original. Consider the isomorph $L$ of $K_{2,4}$ with $x$ and $w$ on one side and $s, t, u, v$ on the other side. Then $D[x w]$ does not cross (the planar drawing) $D[L]$ and so is contained in one of the four faces of $D[L]$. The face of $D[L]$ bounded by swtx is disjoint from $D[J]$. In each of the other three faces, $D[x w]$ must cross $D[s t]$. In two of these three faces, it also crosses exactly one of $D[s u]$ and $D[t v]$. In the third, it crosses both $D[s u]$ and $D[t v]$.

For the moreover, we consider the remaining three types of edges $z_{1} z_{2}: D\left[z_{1}\right]$ and $D\left[z_{2}\right]$ can both be in $R_{1}$; both in $R_{2}$; or one in each. In all three cases for $z_{1}, z_{2}$ and all three cases for the three-cycle $T, D\left[z_{1}\right]$ and $D\left[z_{2}\right]$ are on the same side of $D[T]$. In the event that $D\left[z_{1}\right]$ and $D\left[z_{2}\right]$ are both planarly joined to $D[T]$, Corollary 2.10 applies to show $D\left[z_{1} z_{2}\right]$ does not cross the 3 -cycle.

In the remaining cases, we assume that $D\left[z_{1}\right]$ is not planarly joined to $D[T]$. If $T=$ $s t w$, then Claim 5.3(3) and (5) imply the only possible crossing with $D[T]$ is $D\left[z_{1} w\right]$ crossing $D[s t]$. As $D\left[z_{1} z_{2}\right]$ has either 0 or 2 crossings with $D[s t w]$, but does not cross $D\left[z_{1} w\right]$, the two crossings of $D\left[z_{1} z_{2}\right]$ and $D[T]$ cannot be on $D[w s]$ and $D[w t]$. For $T=s u v$ and $T=t u v$, the edges $z_{1} u$ and $z_{1} v$, respectively, produce analogous results.

We are now prepared for the final part of the proof. For $i=1,2$, let $r_{i}$ be the number of vertices of $D\left[K_{n}\right]$ that are in (the interior of) $R_{i}$.

Let $D^{\prime}$ be the drawing of $K_{n}$ obtained from $D$ by making the following two changes:

(C1) reroute st alongside the path $D[s w t]$, so as to not cross $D[w u]$ and $D[w v]$; and

(C2) reroute $s u$ alongside the path $D[s v u]$ so as to cross $D[v w]$.

We first consider the changes in crossings arising from rerouting st. There are at least $2+r_{2}$ crossing pairs of edges in $D$ that do not cross in $D^{\prime}$ : two from $D[s t]$ crossing $D[w v]$ and $D[w u]$, plus all the crossings of $D[s t]$ from those edges incident with $D[w]$ that cross $D[s t]$. There are at least $r_{2}$ of these latter crossings, as every vertex $z$ such that $D[z]$ is in $R_{2}$ has $D[z w]$ crossing $D[s t]$.

On the other hand, there is a set of at most $r_{1}$ crossing pairs in $D^{\prime}$ that do not cross in $D$. These arise from the the edges joining a vertex drawn in $R_{1}$ to $D[w]$; these might not intersect $D[J]$. Those that do intersect $D[J]$ cross $D[s t]$ and, therefore, yield further savings.

We show that every other edge $z_{1} z_{2}$ has no more crossings in $D^{\prime}$ than it has in $D$.

Case 1: $z_{1}$, say, is in $J$.

In this case, we use Claim 5.3. Items (1), (2), (4), and (5) show that no such edge has more crossings in $D^{\prime}$ than in $D$, except possibly $x w$.

If $D[x w]$ does not cross $D[J]$, then $D^{\prime}[x w]$ also does not cross $D^{\prime}[J-s t]$, as required. If $D[x w]$ crosses $D[J]$, then Claim 5.3(3) implies that $D[x w]$ crosses $D[s t]$. Thus, $D[x w]$ crosses both $D[s u]$ and one of $D[s v]$ and $D[t u]$; in this case, the same is true of $D^{\prime}[x w]$ in $D^{\prime}$, as required.

Case 2: neither $z_{1}$ nor $z_{2}$ is in J. 
If $D\left[z_{1} z_{2}\right]$ crosses the 3 -cycle $D[s t w]$, then the moreover part of Claim 5.3 shows it crosses $D[s t]$. Therefore, it crosses exactly one of $D[s w]$ and $D[w t]$, showing that $D^{\prime}\left[z_{1} z_{2}\right]$ crosses $D^{\prime}[s t]$ and the same one of $D^{\prime}[s w]$ and $D^{\prime}[w t]$. That is, $z_{1} z_{2}$ crosses the same two edges in both drawings, and we are done.

The net result is that the rerouting of st contributes at least $2+\left(r_{2}-r_{1}\right)$ to $\operatorname{cr}(D)-$ $\operatorname{cr}\left(D^{\prime}\right)$.

Now we turn our attention to the changes in crossings from rerouting $s u$. The crossing of $D[s u]$ with $D[t v]$ is replaced by a crossing of $D^{\prime}[s u]$ with $D^{\prime}[v w]$. In addition, $r_{2}$ edges incident with $v$ do not cross $D[s u]$, but cross $D^{\prime}[s u]$, while $r_{1}$ edges incident with $v$ cross $D[s u]$, but do not cross $D^{\prime}[s u]$.

The only additional remark special to this rerouting is the observation that, for $z$ in $R_{1}$, Claim 5.3 implies that if $D[z w]$ crosses the 3-cycle $D[s u v]$, then $z w$ crosses $s u$. This shows that $D^{\prime}[z w]$ also crosses $D^{\prime}[s u v]$ twice, so there are no other "new" crossings.

Therefore, the $s u$ rerouting contributes at least $r_{1}-r_{2}$ to the difference $\operatorname{cr}(D)-$ $\operatorname{cr}\left(D^{\prime}\right)$. Combining this with the contribution from st, we get that $\operatorname{cr}(D)-\operatorname{cr}\left(D^{\prime}\right) \geq$ $\left(2+r_{2}-r_{1}\right)+\left(r_{1}-r_{2}\right)=2$. That is, $\operatorname{cr}\left(D^{\prime}\right) \leq \operatorname{cr}(D)-2$, as required.

(II) $J=\widetilde{\mathbb{K}}_{5}^{5}$.

In this case, there is a homeomorphism $\Theta$ of the sphere to itself that is an involution that restricts to $J$ as, using the labelling in Figure 4: $s \leftrightarrow w ; t \leftrightarrow v$; and $u$ is fixed. This will be helpful at several points in the following discussion. The outline of the argument is the same as for $\widetilde{\mathbb{K}}_{5}^{3}$, but there are some interesting differences.

Let $R_{1}$ be the face of $D[J]$ incident with all three points in $D[\{s, t, u\}]$ (the unbounded face in the diagram) and let $R_{2}$ be the face of $D[J]$ incident with all three points in $D[\{u, v, w\}]$ (note that $R_{2}=\Theta\left(R_{1}\right)$ ).

Claim 5.4. If $z$ is a vertex of $K_{n}$ not in $J$, then $D[z] \in R_{1} \cup R_{2}$.

Proof of Claim 5.4. Suppose $x$ is a vertex of $K_{n}-V(J)$ such that $D[x]$ is not in $R_{1} \cup R_{2}$. Suppose first that $D[x]$ is in the region bounded by the 4-cycle $D[w t s v]$.

The convexity of $D[(J-s)+x]$ and of $D[(J-w)+x]$ imply that $D[x u]$ does not cross the 4-cycles $D[t w v u]$ and $D[s t u v]$, respectively. However, $D[x]$ is not in a face of $D[t w v u] \cup D[s t u v]$ incident with $D[u]$, a contradiction.

The remaining possibility is that $D[x]$ is in the face $F$ that is both distinct from $R_{1}$ and either incident with $D[u t]$ or, symmetrically, incident with $u v$; we assume the former. The convexity of $D[(J-t)+x]$ and $D[(J-v)+x]$ show that $D[x w]$ does not cross the 4-cycles $D[s w v u]$ and $D[s w u t]$, respectively. However, $D[x]$ is not in a face of $D[s w v u] \cup D[s w u t]$ incident with $D[w]$, a contradiction.

We next move to the routings of the edges from a vertex $D[x]$ in $R_{1} \cup R_{2}$ to $D[J]$.

Claim 5.5. If $D[x] \in R_{1}$, then:

(1) $D[x u]$ and $D[x s]$ do not cross $D[J]$;

(2) $D[x v]$ crosses $D[J]$ only on $D[u w]$, and $D[x w]$ crosses $D[J]$ only on $D[s v]$ and $D[t v] ;$ and 
(3) $D[x t]$ either does not cross $D[J]$ or it crosses $D[J]$ precisely on $D[s v], D[s w]$, and $D[s u]$.

Furthermore, if $D[x], D\left[x^{\prime}\right] \in R_{1}$, then $D\left[x x^{\prime}\right] \subseteq R_{1}$.

Proof of Claim 5.5. The convexity of $D[(J-t)+x]$ and $D[(J-u)+x]$ shows $D[x s]$ does not cross the 4-cycles $D[s u v w]$ and $D[s t w v]$, respectively. The convexity of $J-s+x$ shows $x u$ does not cross $J-s$. Also $x u$ does not cross $s u$ by simplicity, so $x u$ does not cross any edge incident with $s$.

The convexity of $J-t+x$ shows $x v$, respectively $x w$, crosses $J-t$ only $u w$, respectively $t v$. Also $x v$, respectively $x w$, does not cross $v t$, respectively $w t$, by simplicity, so $x v$, respectively $x w$, does not cross any edge incident with $t$.

The convexity of $D[(J-s)+x]$ determines the routing of $D[x t]$, except with respect to $D[s]$, leaving the two options described.

For the furthermore conclusion, $D[x]$ and $D\left[x^{\prime}\right]$ are planarly joined to the 3 -cycle $D[s v u]$. Corollary 2.10 shows that $D\left[x x^{\prime}\right]$ is disjoint from $D[s v u]$. In the same way, $D\left[x x^{\prime}\right]$ is disjoint from $D[s w u]$, and $D[t v u]$. Thus, $D\left[x x^{\prime}\right]$ can only cross $D[J]$ on $D[s t]$. However, letting $\times$ denote the crossing of $D[s u]$ with $D[t v], D\left[x x^{\prime}\right]$ must cross the 3 -cycle $D[s t \times]$ an even number of times and it can only cross it on $D[s t]$, which is impossible.

The homeomorphism $\Theta$ implies a completely symmetric statement when $x \in R_{2}$. We provide it here for ease of reference.

Claim 5.6. If $D[x] \in R_{2}$, then, in $D[J+x]$ :

(1) $D[x u]$ and $D[x w]$ do not cross $D[J]$;

(2) $D[x t]$ crosses $D[J]$ only on $D[u s]$, and $D[x s]$ crosses $D[J]$ only on $D[t w]$ and $D[t v] ;$ and

(3) $D[x v]$ either does not cross $D[J]$ or it crosses $D[J]$ precisely on $D[w t], D[w s]$, and $D[w u]$.

Furthermore, if $D[x], D\left[x^{\prime}\right] \in R_{2}$, then $D\left[x x^{\prime}\right] \subseteq R_{2}$.

Using the homeomorphism $\Theta$, we may choose the labelling of $J$ so that the number $r_{1}$ of vertices of $D\left[K_{n}\right]$ drawn in $R_{1}$ is at most the number $r_{2}$ drawn in $R_{2}$.

Our next claim was somewhat surprising to us in the strength of its conclusion.

Claim 5.7. If there is a vertex $x$ of $K_{n}-V(J)$ such that $D[x] \in R_{1}$ and $D[x t]$ crosses $D[s v], D[s w]$, and $D[s u]$, then there is a drawing $D^{\prime}$ of $K_{n}$ such that $\operatorname{cr}\left(D^{\prime}\right) \leq \operatorname{cr}(D)-4$ and, if $n$ is even, $\operatorname{cr}\left(D^{\prime}\right) \leq \operatorname{cr}(D)-5$.

Proof of Claim 5.7. Choose such an $x$ so that $D[x t]$ crosses $D[s v], D[s w]$, and $D[s u]$ and such that, among all such $x$, the crossing of $D[x t]$ with $D[s v]$ is as close to $D[s]$ on $D[s v]$ as possible. Let $\Delta$ be the closed disc bounded by the 3-cycle $D[s x t]$ that does not contain the vertices $D[\{v, u, w\}]$.

If there is a vertex $y$ of $K_{n}$ such that $D[y]$ is in the interior of $\Delta$, then $D[y]$ is in the face of $D[J+x]$ contained in $\Delta$ and incident with $D[s x]$. However, the convexity in $D$ of $(J-\{u, w\})+\{x, y\}$ implies $D[y t]$ crosses $D[s v]$ closer to $s$ in $D[s v]$ than $D[x t]$ does, contradicting the choice of $x$. Therefore, no vertex of $D\left[K_{n}\right]$ is in $\Delta$. 
The drawing $D^{\prime}$ is obtained from $D$ by rerouting $x t$ to go alongside the path $D[x s t]$, on the side not in $\Delta$. (That is, $D[x t]$ is pushed to the other side of $D[s]$.)

The hardest part of the analysis of the crossings of $D^{\prime}$ compared to $D$ is determining what happens to an edge of $D\left[K_{n}\right]$ that crosses $D[s t]$. No edge of $D[J]$ crosses $D[s t]$. Claims 5.5 and 5.6 imply that: no edge from a vertex in $R_{1} \cup R_{2}$ to a vertex in $D[J]$ crosses $D[s t]$; and no edge with both incident vertices in the same one of $R_{1}$ or $R_{2}$ crosses $D[s t]$. Thus, the only possible crossing of $D[s t]$ is by an edge $D[y z]$, with $D[y] \in R_{1}$ and $D[z] \in R_{2}$.

Because of the routing of $D[s z], D[y z]$ cannot also cross $D[x s]$. Therefore, $D[y z]$ also crosses $D[x t]$. It follows that such an edge has the same number of crossings of $x t$ in both $D$ and $D^{\prime}$. Therefore, any edge that crosses $D[x s]$ crosses $D[x t]$ and so has the same number of crossings with $D[x t]$ and $D^{\prime}[x t]$.

The only changes then are in the number of crossings of $D[x t]$ with edges incident with $D[s]$ and the number of crossings with $D[J]$. There are 3 fewer of the latter. From $R_{1}$ to $D[s]$, there are at most $r_{1}-1$ crossings of $D^{\prime}[x t]$. From $R_{2}$ to $D[s]$, we have lost $r_{2}$ crossings of $D[x t]$. Thus, $D^{\prime}$ has at least $\left(r_{2}-\left(r_{1}-1\right)\right)+3=\left(r_{2}-r_{1}\right)+4$ fewer crossings than $D$. This proves the first conclusion.

Since $n=5+r_{1}+r_{2}$, if $n$ is even, then $r_{1} \neq r_{2}$ and, therefore, $r_{2}-r_{1} \geq 1$. In this case $D^{\prime}$ has at least 5 fewer crossings, as claimed.

It follows from Claim 5.7 that we may assume that, for $D[x] \in R_{1}, D[x t]$ is disjoint from $D[J]$. Combining this with the other information from Claim 5.5, we may assume the following property.

$\mathbf{R}_{1}$ Assumption: If $D[x] \in R_{1}$, then $D[x]$ is planarly joined to $D[J-w]$.

Let $D^{\prime}$ be obtained from $D$ by rerouting $D[t v]$ on the other side of the path $D[t s v]$. There are two claims that complete the proof of Theorem 5.1. The first, similar to Claim 5.7, shows that there are at least 2 fewer crossings in $D^{\prime}$ (3 if $n$ is even). The second shows that $D^{\prime}$ satisfies the hypotheses of Theorem 5.1. Therefore, there is a third drawing $D^{\prime \prime}$ with at least two fewer crossings than $D^{\prime}$, as required.

Claim 5.8. $\operatorname{cr}\left(D^{\prime}\right) \leq \operatorname{cr}(D)-\left(\left(r_{2}-r_{1}\right)-2\right)$.

Proof of Claim 5.8. The proof is very similar to that of Claim 5.7. The main point is to see that no edge $e$ can have $D[e]$ cross both $D[t s]$ and $D[s v]$. Let $x$ be incident with $e$. If $D[x] \in R_{2}$, then the routing of $D[x s]$ is known; since $D[x s]$ does not cross $D[e]$, the crossings of $D[e]$ with $D[t s]$ and $D[s v]$ are joined by an arc of $D[e]$ outside $D[s t u v]$. But then $D[e]$ has both ends in $J \cup R_{2}$. This contradicts Claim 5.6, so $D[x] \notin R_{2}$.

Likewise, Claim 5.5 shows $D[x] \notin R_{1}$ and clearly $e$ is not in $J$. Therefore, there is no such $e$.

It is now easy to see that there are $\left(r_{2}-r_{1}\right)+2$ fewer crossings of $D^{\prime}[t v]$ with edges incident with $s$ than there are of $D[t v]$. All other crossings of $D^{\prime}[t v]$ pair off with crossings of $D[t v]$.

Finally, we show that the drawing $D^{\prime}$ satisfies the hypotheses of Theorem 5.1. It is routine to verify that $D^{\prime}[J]$ is $\widetilde{\mathbb{K}}_{5}^{3}$. Now let $N$ be a $K_{5}$ in $K_{n}$ such that $N \cap J$ has 3 or 4 vertices.

If any of $s, t, v$ is not in $N$, then $D^{\prime}[N]$ is homeomorphic to $D[N]$ and so is convex. Thus, we may assume $s, t, v$ are all in $N$. 


\section{Case 1: $N \cap J$ has four vertices.}

In this case, there is a vertex $x$ not in $J$ such that $N$ is either $(J-w)+x$ or $(J-u)+x$. If $D[x]$ is in $R_{1}$, then the routings are determined and we can see by inspection that $D^{\prime}[N]$ is, respectively, the $K_{5}$ with 1 crossing or the convex $K_{5}$ with 3 crossings.

If $D[x] \in R_{2}$, then Claim 5.6 shows that $D^{\prime}[(J-w)+x]$ is the $K_{5}$ with one crossing. However, Claim 5.6(3) shows $D^{\prime}[(J-u)+x]$ has two possibilities for $x v$, depending on which way around $w$ it goes. If it crosses both $w t$ and $w s$, then the drawing is the natural one. The alternative is to reroute it to not cross $D^{\prime}[N-x]$. In this case, Observation 2.8 shows $D^{\prime}[N]$ is convex.

Case 2: $N \cap J$ has 3 vertices.

In this case $N=(J-\{u, w\})+\{x, y\}$. Since $D[x], D[y] \in R_{1} \cup R_{2}$, they are both on the same side of $D[s t v]$. The routings from either to $D[J]$ are determined by Claims 5.5 and 5.6 and the assumption following the proof of Claim 5.7. Only when $D[x]$ and $D[y]$ are in different ones of $R_{1}$ and $R_{2}$ is it possible that $D[x y]$ crosses $D[s t v]$.

We consider the three possibilities for $D[x]$ and $D[y]$.

Subcase 2.1: $D[x] \in R_{1}$ and $D[y] \in R_{2}$.

All routings in $D^{\prime}[N]$ are determined except for $D[x y]$. The 4-cycle $D[x v y t]$ is uncrossed in $D[N-x y]$. As $D$ is a drawing, $D[x y]$ does not cross $D[x v y t]$. Therefore, either $D[x v y t]$ or $D[x v y]$ is a face of $D^{\prime}[N]$, showing $D^{\prime}[N]$ is convex.

Subcase 2.2: $D[x]$ and $D[y]$ are both in $R_{2}$.

Since $D[x]$ and $D[y]$ are both planarly joined to $D^{\prime}[s t v]$ and $D[x y]$ does not cross $D^{\prime}[s t v], D^{\prime}[s t v]$ bounds a face of $D^{\prime}[N]$. Thus, $D^{\prime}[N]$ is convex.

Subcase 2.3: $D[x], D[y]$ are both in $R_{1}$.

Suppose $D^{\prime}[N]$ is not convex. Then Corollary 2.5(a) implies there is a 3-cycle $T$ in $N$ such that the two vertices $z, z^{\prime}$ of $N$ not in $T$ are in different faces of $D^{\prime}[T]$ and both $D^{\prime}[T+z]$ and $D^{\prime}\left[T+z^{\prime}\right]$ are crossing $K_{4}$ 's.

Since both $x$ and $y$ are in the same face of $D^{\prime}[s t v], T \neq s t v$. If $a \in\{s, t, v\}$, then the routings of the edges from $x$ and $y$ to stv show that the two vertices in $\{s, t, v\} \backslash\{a\}$ are on the same side of $D^{\prime}[x y a]$, so $x y a \neq T$. The only remaining possibility is that $T$ has $x$, say, and two of $s, t, v$.

Claim 5.9. The 3 -cycle $D^{\prime}[t v x]$ has no convex side.

Proof of Claim 5.9. In the alternative, $T$ is either stx or $s v x$. These two situations are very similar, so we treat only stx, leaving the completely analogous argument for $s v x$ to the reader. Our strategy is to show that assuming that $s t x$ has no convex side in $D^{\prime}$ implies that $t v x$ has no convex side in $D^{\prime}$ either.

The vertices $v$ and $y$ are on different sides of $D^{\prime}[s t x]$ and $D[v t]$ crosses $D[s x]$, showing that the side of $D^{\prime}[s t x]$ containing $D[v]$ is not convex. The edge $D[s x]$ also shows that the side of $D^{\prime}[t v x]$ containing $D[s]$ is not convex.

Likewise, there is an edge $e$ incident with $y$ to one of $s, t$, and $x$ such that $D[e]$ crosses $D^{\prime}[s t x]$. Notice that $D[x y]$ does not cross $D[x s]$ and $D[x t]$ by definition of drawing and $D[x y]$ does not cross $D[s t]$ by the $R_{1}$ Assumption. Therefore, $D[x y]$ does not cross $D[s t]$ and we conclude that $D[x y]$ does not cross $D[s t x]$. 
Next suppose that that $D[y t]$ crosses $D[x s]$. The $R_{1}$ Assumption shows that $D[y t]$ does not cross $D[s t v]$ and so $D[y t]$ crosses $D[v x]$. Therefore, this side of $D^{\prime}[t v x]$ is also not convex. Combined with the second paragraph of this proof, $D^{\prime}[t v x]$ is not convex.

In the final case, $D[y s]$ crosses $D[x t]$. As we traverse $D[y s]$ from $D[y]$, there is the crossing with $D[x t]$. A point of $D[y s]$ just beyond this crossing is on the other side of $D[t v x]$ from both $y$ and $s$.

The edge $D[y v]$ is contained on the same side of the 3-cycle $D[s t y]$ as $D[v]$. Therefore, $D[y v]$ must also cross $D[x t]$, showing that the $D[y]$-side of $D[t v x]$ is also not convex, as required.

Notice that $D[y]$ is in one side of $D^{\prime}[t v x]$ and $D[s]$ is on the other. Since $s \notin\{t, v, x, y\}$, $D[\{t, v, x, y\}]$ and $D^{\prime}[\{t, v, x, y\}]$ are homeomorphic. Thus, the side of $D[t v x]$ that contains $D[y]$ is not convex in $D$.

On the other hand, we know that, in $D, D[w]$ is on the other side of $D[t v x]$ from $D[y]$. However, Claim 5.5(2) shows that $D[w x]$ crosses $D[t v]$. This shows that the side of $D[t v x]$ containing $D[w]$ is not convex. Combined with the preceding paragraph, the $K_{5}$ induced by $t, v, w, x, y$ is not convex in $D$, contradicting the hypothesis of the theorem. This completes the proof of Subcase 2.3 and the theorem.

It would be significant progress to prove some analogue of Theorem 5.1 with a weaker hypothesis on extensions $J$. Indeed, one might expect that no hypothesis beyond the existence of $J$ is required, as is easily verified for $n=7$ (Lemmas 7.5 and 7.7 [21] prove this for $K_{7}$ as a simple consequence of the theory developed).

Suppose a drawing $D$ of $K_{8}$ has a non-convex $K_{5}$. This $K_{5}$ is in three different $K_{7}$ 's, each having at least 11 crossings. Lemma 5.10 below shows $D$ has at least 20 crossings, in agreement with Aichholzers's computations.

Lemma 5.10. Let $n$ be an integer, $n \geq 4$, and let $D$ be a drawing of $K_{n}$. Then $(n-4) \operatorname{cr}(D)=\sum_{v \in V\left(K_{n}\right)} \operatorname{cr}(D-v)$.

A similar argument shows that a non-convex drawing of $K_{9}$ cannot have 34 crossings. Let $J$ be any non-convex $K_{5}$ in a drawing $D$ of $K_{9}$ having 34 crossings. Then $J$ is contained in four $K_{8}$ 's in the $K_{9}$. The preceding paragraph shows each of these $K_{8}$ 's has at least 20 crossings. Lemma 5.10 and the assumption that $D$ has only 34 crossings shows that the five remaining $K_{8}$ 's are optimal and hence convex. Thus, $J$ is the only non-convex $K_{5}$ in $D$ and so the hypothesis of Theorem 5.1 trivially holds.

The hypothesis of Theorem 5.1 is stronger than we would like and stronger than needed for the preceding argument for $K_{8}$. It is not so strong, however, as to force a single nonconvex $K_{5}$ in a drawing. For example, we have a drawing of $K_{8}$ - a modified $T C_{8}-$ having two different non-convex $K_{5}$ 's and satisfying the hypotheses of Theorem 5.1.

\section{Questions and conjectures}

We conclude with a few questions and conjectures.

1. In Section 1 we presented a table with the convexity hierarchy. One obvious omission is a forbidden drawing characterization of when an h-convex drawing is f-convex. We pointed out that $T C_{8}$ is one example of h-convex that is not f-convex. Rerouting some of the edges between the central and outer crossing $K_{4}$ 's produces a few more examples. 
Question 6.1. Is there a characterization by forbidden subdrawings of those h-convex drawings of $K_{n}$ that are f-convex?

2. The deficiency $\delta(D)$ of a drawing $D$ of $K_{n}$ is the number $\operatorname{cr}(D)-H(n)$. The drawing $D$ has the natural deficiency property if, for every vertex $v$ of $K_{n}, \delta(D-v) \leq 2 \delta(D)$. If the Hill Conjecture is true for $n=2 k+1$, then every drawing of $K_{2 k}$ has the natural deficiency property. We prove this in the Appendix.

Conjecture 6.2. For every $k \geq 2$, every simple drawing of $K_{2 k}$ has the natural deficiency property.

This seems to be an interesting weakening of the Hill Conjecture; it came up tangentially in the proof that $\operatorname{cr}\left(K_{13}\right)>217$ [20].

3. Pach, Solymosi, and Tóth [23] proved that, for each positive integer $r$, there is an $N(r)=\mathrm{O}\left(2^{r^{8}}\right)$ such that, for every $n \geq N(r)$, every drawing $D$ of $K_{n}$ contains either the natural $K_{r}$ or the Harborth $K_{r}$ [15]. If $D$ is convex, then it must be the natural $K_{r}$.

Question 6.3. Can the $\mathrm{O}\left(2^{r^{8}}\right)$ bound be improved for convex drawings?

4. The big question is: Is every optimal drawing of $K_{n}$ convex? While we believe this is quite conceivable, Ramsey theory suggests other possibilities. Hedging our bets, we have the following conjecture.

Conjecture 6.4. Exactly one of the following holds:

(a) for all $n \geq 5$, no optimal drawing of $K_{n}$ contains $\widetilde{\mathbb{K}}_{5}^{5}$; and

(b) for any $p \geq 1$ and any drawing $D$ of $K_{p}$, there is some $n \geq p$ and an optimal drawing of $K_{n}$ (or at least one with at most $H(n)$ crossings) that contains $D\left[K_{p}\right]$.

\section{ORCID iDs}

Alan Arroyo (D) https://orcid.org/0000-0003-2401-8670
R. Bruce Richter (iD https://orcid.org/0000-0002-1444-9699

\section{References}

[1] B. Ábrego, O. Aichholzer, S. Fernández-Merchant, P. Ramos, and B. Vogtenhuber, Nonshellable drawings of $k_{n}$ with few crossings, in: 26th Annual Canadian Conference on Computational Geometry CCCG 2014, 2014 pp. 46-51, https://www.cccg.ca/ proceedings/2014/.

[2] O. Aichholzer, Rotation systems with specific drawings of $K_{5}$, in preparation.

[3] O. Aichholzer, T. Hackl, A. Pilz, P. Ramos, V. Sacristán and B. Vogtenhuber, Empty triangles in good drawings of the complete graph, Graphs Comb. 31 (2015), 335-345, doi:10.1007/ s00373-015-1550-5.

[4] O. Aichholzer, T. Hackl, A. Pilz, G. Salazar, and B. Vogtenhuber, Deciding monotonicity of good drawings of the complete graph, in: XVI Spanish Meeting on Computational Geometry (EGC 2015), 2015 pp. 33-36, http: / /www. ist.tugraz . at/cpgg/publications. php. 
[5] A. Arroyo, D. McQuillan, R. B. Richter and G. Salazar, Levi's lemma, pseudolinear drawings of $K_{n}$, and empty triangles, J. Graph Theory 87 (2018), 443-459, doi:10.1002/jgt.22167.

[6] A. Arroyo, R. B. Richter and M. Sunohara, Extending drawings of complete graphs into arrangements of pseudocircles, SIAM J. Discrete Math. 35 (2021), 1050-1076, doi:10.1137/ $20 \mathrm{~m} 1313234$.

[7] J. Balogh, B. Lidický and G. Salazar, Closing in on Hill's conjecture, SIAM J. Discrete Math. 33 (2019), 1261-1276, doi:10.1137/17m1158859.

[8] I. Bárány and Z. Füredi, Empty simplices in Euclidean space, Can. Math. Bull. 30 (1987), 436-445, doi:10.4153/cmb-1987-064-1.

[9] I. Bárány and P. Valtr, Planar point sets with a small number of empty convex polygons, Stud. Sci. Math. Hung. 41 (2004), 243-266, doi:10.1556/sscmath.41.2004.2.4.

[10] L. Beineke and R. Wilson, The early history of the brick factory problem, Math. Intell. 32 (2010), 41-48, doi:10.1007/s00283-009-9120-4.

[11] W. E. Bonnice, On convex polygons determined by a finite planar set, Am. Math. Mon. 81 (1974), 749-752, doi:10.2307/2319566.

[12] P. Erdős and G. Szekeres, On some extremum problems in elementary geometry, Ann. Univ. Sci. Budap. Rolando Eötvös, Sect. Math. 3-4 (1961), 53-62, https : / / users . renyi . hu/ p_erdos/Erdos.html.

[13] R. K. Guy, Crossing numbers of graphs, in: Y. Alavi, D. R. Lick and A. T. White (eds.), Graph Theory and Applications, Springer Berlin Heidelberg, Berlin, Heidelberg, 1972 pp. 111-124, doi: $10.1007 / \mathrm{bfb} 0067363$.

[14] F. Harary and A. Hill, On the number of crossings in a complete graph, Proc. Edinb. Math. Soc., II. Ser. 13 (1963), 333-338, doi:10.1017/s0013091500025645.

[15] H. Harborth, Empty triangles in drawings of the complete graph, Discrete Math. 191 (1998), 109-111, doi:10.1016/s0012-365x(98)00098-3.

[16] H. Harborth and I. Mengersen, Drawings of the complete graph with maximum number of crossings, in: Proceedings of the Twenty-Third Southeastern International Conference on Combinatorics, Graph Theory, and Computing (Boca Raton, FL, 1992), Congr. Numer. 88, pp. 225-228, 1992.

[17] D. J. Kleitman, A note on the parity of the number of crossings of a graph, J. Comb. Theory, Ser. B 21 (1976), 88-89, doi:10.1016/0095-8956(76)90032-0.

[18] F. Levi, Die Teilung der projektiven Ebene durch Gerade oder Pseudogerade, Ber. Math.-Phys. Kl. Sächs. Akad. Wiss. Leipzig 78 (1926), 256-267.

[19] J. Matousek, Intersection graphs of segments and $\exists \mathbb{R}^{2}$, 2014, arXiv:1406.2636 [math.CO].

[20] D. McQuillan, S. Pan and R. B. Richter, On the crossing number of $K_{13}, J$. Comb. Theory, Ser. B 115 (2015), 224-235, doi:10.1016/j.jctb.2015.06.002.

[21] D. McQuillan and R. B. Richter, On the crossing number of $K_{n}$ without computer assistance, J. Graph Theory 82 (2016), 387-432, doi:10.1002/jgt.21908.

[22] N. E. Mnëv, The universality theorems on the classification problem of configuration varieties and convex polytopes varieties, in: Topology and Geometry-Rohlin Seminar, Springer, Berlin, volume 1346 of Lecture Notes in Math., pp. 527-543, 1988, doi:10.1007/bfb0082792.

[23] J. Pach, J. Solymosi and G. Tóth, Unavoidable configurations in complete topological graphs, Discrete Comput. Geom. 30 (2003), 311-320, doi:10.1007/s00454-003-0012-9. 
[24] A. Suk, On the Erdős-Szekeres convex polygon problem, J. Am. Math. Soc. 30 (2017), 10471053, doi:10.1090/jams/869.

[25] U. Wagner, On a geometric generalization of the upper bound theorem, in: 2006 47th Annual IEEE Symposium on Foundations of Computer Science (FOCS'O6), 2006 pp. 635-645, doi: 10.1109/focs.2006.53.

\section{Appendix}

Here we prove the claim made in the discussion about the natural deficiency property, related to Conjecture 6.2: if the Hill Conjecture is true for $n=2 k+1$, then every drawing of $K_{2 n}$ has the natural deficiency property.

Let $D$ be a simple drawing of $K_{2 n}$, let $v$ be any vertex of $K_{2 n}$, and let $r(v)$ denote the total number of crossings in $D$ involving edges incident with $v$. Duplicating $v$ to get the drawing $D+v$ of $K_{2 n+1}$, we get $\operatorname{cr}(D+v)=\operatorname{cr}(D)+W(2 n-1)+r(v)$, where $W(m)=\left\lfloor\frac{m}{2}\right\rfloor\left\lfloor\frac{m-1}{2}\right\rfloor$ is the smallest number of crossings in a drawing of $K_{2, m}$ such that the vertices on the 2 -side have the same clockwise rotation of the $m$ remaining vertices.

By assumption, $\operatorname{cr}(D+v) \geq H(2 n+1)$, so $H(2 n+1) \leq \operatorname{cr}(D)+W(2 n-1)+r(v)$. On the other hand, arithmetic shows that $H(2 n+1)=H(2 n)+W(2 n-1)+(H(2 n)-$ $H(2 n-1))$. Therefore,

$$
\begin{aligned}
2 H(2 n)+W(2 n-1)-H(2 n-1) & =H(2 n+1) \\
& \leq \operatorname{cr}(D)+W(2 n-1)+r(v) .
\end{aligned}
$$

Cancelling the common $W(2 n-1)$ and rearranging yields

$$
r(v)+H(2 n-1) \geq 2 H(2 n)-\operatorname{cr}(D) .
$$

Inequality (A.1) implies

$$
\begin{aligned}
\delta(D-v) & =\operatorname{cr}(D-v)-H(2 n-1) \\
& =\operatorname{cr}(D)-r(v)-H(2 n-1) \\
& \leq 2 \operatorname{cr}(D)-2 H(2 n) \\
& =2 \delta(D),
\end{aligned}
$$

as claimed. 